\title{
Hardy-type inequalities related to degenerate elliptic differential operators
}

\author{
LORENZO D’AMBROSIO
}

\begin{abstract}
We prove some Hardy-type inequalities related to quasilinear secondorder degenerate elliptic differential operators $L_{p} u:=-\nabla_{L}^{*}\left(\left|\nabla_{L} u\right|^{p-2} \nabla_{L} u\right)$. If $\phi$ is a positive weight such that $-L_{p} \phi \geq 0$, then the Hardy-type inequality$$
c \int_{\Omega} \frac{|u|^{p}}{\phi^{p}}\left|\nabla_{L} \phi\right|^{p} d \xi \leq \int_{\Omega}\left|\nabla_{L} u\right|^{p} d \xi \quad\left(u \in \mathscr{C}_{0}^{1}(\Omega)\right)
$$

holds. We find an explicit value of the constant involved, which, in most cases, results optimal. As particular case we derive Hardy inequalities for subelliptic operators on Carnot Groups.
\end{abstract}

Mathematics Subject Classification (2000): 35H10 (primary); 22E30, 26D10, 46E35 (secondary).

\section{Introduction}

An $N$-dimensional generalization of the classical Hardy inequality is the following

$$
c \int_{\Omega}|u|^{p} w^{-p} d x \leq \int_{\Omega}|\nabla u|^{p} d x, \quad u \in \mathscr{C}_{0}^{1}(\Omega),
$$

where $p>1, \Omega \subset \mathbb{R}^{N}$ and the weight $w$ is, for instance, $w:=|x|$ or $w(x):=$ $\operatorname{dist}(x, \partial \Omega)$ (see for instance $[5,10,23]$ and the references therein).

A lot of efforts have been made to give explicit values of the constant $c$, and even more, to find its best value $c_{n, p}$ (see e.g. [5, 10, 23, 24, 31, 40, 41, 42]).

The preeminent rôle of the Hardy inequality in the study of linear and nonlinear partial differential equations is well-known. For instance, let us consider the linear initial value problem

$$
\begin{cases}u_{t}-\Delta u=\lambda \frac{u}{|x|^{2}}, & \left.x \in \mathbb{R}^{n}, \quad n \geq 3, \quad t \in\right] 0, T[, \quad \lambda \in \mathbb{R}, \\ u(x, 0)=u_{0}(x), \quad x \in \mathbb{R}^{n}, & u_{0} \in L^{2}\left(\mathbb{R}^{n}\right), \quad u_{0}>0 .\end{cases}
$$

Pervenuto alla Redazione il 9 febbraio 2005 e in forma definitiva il 3 agosto 2005. 
The problem (1.2) has a solution if and only if $\lambda \leq\left(\frac{n-2}{2}\right)^{2}=c_{n, 2}$ (see [3] for more details). In the last years this result has been extended in several directions, see e.g. $[9,12,28,32,45,46,49]$.

In the Heisenberg-group setting, Garofalo and Lanconelli in [29], Niu, Zhang and Wang in [47] and the author in [19] proved, among other results, the following Hardy-type inequality related to the sub-Laplacian $\Delta_{H}$ on the Heisenberg-group $\mathbb{H}^{n}$ :

$$
c \int_{\mathbb{H}^{n}} \frac{u^{2}}{\rho^{2}} \psi_{H}^{2} d \xi \leq \int_{\mathbb{H}^{n}}\left|\nabla_{H} u\right|^{2} d \xi, \quad u \in \varnothing_{0}^{1}\left(\mathbb{H}^{n} \backslash\{0\}\right)
$$

where $\nabla_{H}$ denotes the vector field associated to the real part of the Kohn Laplacian $\left(\Delta_{H}=\nabla_{H} \cdot \nabla_{H}\right), \rho$ and $\psi_{H}$ are respectively a suitable distance from the origin and a weight function such that $0 \leq \psi_{H} \leq 1$.

Recently, in [32], it has been pointed out that the analogue problem of (1.2) involving the sub-Laplacian $\Delta_{H}$, namely

$$
\begin{cases}u_{t}-\Delta_{H} u=\lambda \psi_{H}^{2} \frac{u}{\rho^{2}} & \text { on } \left.\mathbb{R}^{2 n+1} \times\right] 0, T[, \quad \lambda \in \mathbb{R}, \\ u(\cdot, 0)=u_{0}(\cdot) & \text { on } \mathbb{R}^{2 n+1}, \quad u_{0} \in L^{2}\left(\mathbb{R}^{2 n+1}\right), \quad u_{0}>0,\end{cases}
$$

has a positive solution if and only if $\lambda \leq c_{b, H}$, where $c_{b, H}$ is the best constant in (1.3).

Similar results have been established for equations involving the BaouendiGrushin-type operators $\Delta_{\gamma}:=\Delta_{x}+|x|^{2 \gamma} \Delta_{y}=\nabla_{\gamma} \cdot \nabla_{\gamma}$ (see [37]).

Recently, in [21] Mitidieri, Pohozaev and the author, among other results, found some conditions on the functions $u$ and $f$ that assure the positivity of the solutions of the partial differential inequalities $-L u \geq f(\xi, u)$ on $\mathbb{R}^{N}$. Here $L$ is a quite general linear second-order differential operator, namely, $L u:=-\nabla_{L}^{*} \cdot \nabla_{L} u$, where $\nabla_{L}$ is a general vector field. This class of operators include all previously cited operators as well as the sub-Laplacian on Carnot groups.

Having in mind some extensions of the above results to the setting of secondorder linear degenerate (or singular) partial differential operators, it appears that an important step towards this programme is to establish some fundamental inequalities of Hardy-type.

In this paper we shall prove some Hardy-type inequalities associated to the quasilinear operators

$$
L_{p} u:=-\nabla_{L}^{*}\left(\left|\nabla_{L} u\right|^{p-2} \nabla_{L} u\right) \quad(p>1) .
$$

Our principal result can be roughly described as follows: if $\phi: \Omega \rightarrow \mathbb{R}$ is any positive weight, for any $u \in \mathscr{C}_{0}^{1}(\Omega)$ we have

$$
c \int_{\Omega} \frac{|u|^{p}}{\phi^{p}}\left|\nabla_{L} \phi\right|^{p} d \xi \leq \int_{\Omega}\left|\nabla_{L} u\right|^{p} d \xi
$$

provided $-L_{p} \phi \geq 0$. 
For this goal we shall mainly use a technique developed in [18, 19, 44]. An interesting outcome of this approach is that, in several cases, one can easily obtain the best constant. Furthermore, our main results represent a generalization of some results contained in $[4,5]$. Indeed, in those papers the authors deal with a very special case, the usual Euclidean case where $\phi$ is a particular power of the Euclidean distance from a given surface, whereas, in our approach, $\nabla_{L}$ can be any quite general vector field and $\phi$ any positive weight, the generality of this approach being an important strength. It is, in fact, to remark that this unifying method allows, specializing the choice of $\phi$, to obtain almost all the fundamental Hardy inequalities known in Euclidean and subelliptic settings as well as to yield new Hardy-type inequalities. Moreover, let us to stress that our only hypothesis $-L_{p} \phi \geq 0$ plays a relevant role in order to establish that the best constant is not achieved.

We pay particular attention to the following special cases of $L_{p}$ : the Grushin type operators, the Heisenberg-Greiner operators and the sub-Laplacian on Carnot groups (see Section 3). Specializing the function $\phi$, we get more concrete Hardytype inequalities for these operators with explicit values of the constants involved, which result the best possible in almost all the considered cases.

\section{Main results}

The aim of this section is to present some preliminary results and derive some Hardy-type inequalities related to a general vector field.

In this paper $\nabla$ stands for the usual gradient in $\mathbb{R}^{N}$. We indicate with $I_{k}$ and with $|\cdot|$ respectively the identity matrix of order $k$ and the Euclidean norm.

Let $\mu:=\left(\mu_{i j}\right), i=1, \ldots, l, j=1, \ldots, N$ be a matrix with continuous entries $\mu_{i j} \in \mathscr{C}\left(\mathbb{R}^{N}\right)$. Let $X_{i}(i=1, \ldots, l)$ be defined as

$$
X_{i}:=\sum_{j=1}^{N} \mu_{i j}(\xi) \frac{\partial}{\partial \xi_{j}}
$$

and let $\nabla_{L}$ be the vector field defined by

$$
\nabla_{L}:=\left(X_{1}, \ldots, X_{l}\right)^{T}=\mu \nabla .
$$

Assuming that for $i=1, \ldots, l$ and $j=1, \ldots, N$ the derivative $\frac{\partial}{\partial \xi_{j}} \mu_{i j} \in \mathscr{C}\left(\mathbb{R}^{N}\right)$, we set

$$
X_{i}^{*}:=-\sum_{j=1}^{N} \frac{\partial}{\partial \xi_{j}} \mu_{i j}(\xi) .
$$

the formal adjoint of $X_{i}$ and $\nabla_{L}^{*}:=\left(X_{1}^{*}, \ldots, X_{l}^{*}\right)^{T}$.

For any vector field $h=\left(h_{1}, \ldots, h_{l}\right)^{T} \in \mathscr{C}^{1}\left(\Omega, \mathbb{R}^{l}\right)$, we shall use the following notation

$$
\operatorname{div}_{L}(h):=\operatorname{div}\left(\mu^{T} h\right)
$$


that is

$$
\operatorname{div}_{L}(h)=-\sum_{i=1}^{l} X_{i}^{*} h_{i}=-\nabla_{L}^{*} \cdot h
$$

In what follows $L$ stands for the linear second-order differential operator defined by

$$
L:=\operatorname{div}_{L}\left(\nabla_{L}\right)=-\sum_{i=1}^{l} X_{i}^{*} X_{i}=-\nabla_{L}^{*} \cdot \nabla_{L}
$$

and for $p>1$, with $L_{p}$ we denote the quasilinear operator

$$
L_{p}(u):=\operatorname{div}_{L}\left(\left|\nabla_{L} u\right|^{p-2} \nabla_{L} u\right)=-\sum_{i=1}^{l} X_{i}^{*}\left(\left|\nabla_{L} u\right|^{p-2} X_{i} u\right)=-\nabla_{L}^{*} \cdot\left(\left|\nabla_{L} u\right|^{p-2} \nabla_{L} u\right) .
$$

Example 2.1. Let $l<N$ be a positive natural number and let $\mu^{l}$ be the matrix defined as

$$
\mu^{l}:=\left(\begin{array}{ll}
I_{l} & 0
\end{array}\right) .
$$

The corresponding vector field $\nabla^{l}$ results to be the usual gradient acting only on the first $l$ variables $\nabla^{l}=\left(\frac{\partial}{\partial \xi_{1}}, \frac{\partial}{\partial \xi_{2}}, \ldots, \frac{\partial}{\partial \xi_{l}}\right)$. It is clear that $\nabla^{N}=\nabla$. The corresponding quasilinear operator $L_{p}$ is the usual $p$-Laplacian acting on the first $l$ variables of $\mathbb{R}^{N}$.

Example 2.2. (Baouendi-Grushin type operator) Let $\mathbb{R}^{N}$ be splitted in $\xi=(x, y) \in$ $\mathbb{R}^{n} \times \mathbb{R}^{k}$. Let $\gamma \geq 0$ and let $\mu$ be the following matrix

$$
\left(\begin{array}{ccc}
I_{n} & 0 & \\
0 & |x|^{\gamma} & I_{k}
\end{array}\right)
$$

The corresponding vector field is $\nabla_{\gamma}=\left(\nabla_{x},|x|^{\gamma} \nabla_{y}\right)$ and the linear operator $L$ is the so-called Baouendi-Grushin operator $L=\Delta_{x}+|x|^{2 \gamma} \Delta_{y}$.

Notice that if $k=0$ or $\gamma=0$, then $L$ and $L_{p}$ coincide respectively with the usual Laplacian operator and $p$-Laplacian operator.

Example 2.3. (Heisenberg gradient) Let $\xi=(x, y, t) \in \mathbb{R}^{n} \times \mathbb{R}^{n} \times \mathbb{R}=\mathbb{H}^{n}(=$ $\mathbb{R}^{N}$ ) and let $\mu$ be defined as

$$
\left(\begin{array}{ccc}
I_{n} & 0 & 2 y \\
0 & I_{n} & -2 x
\end{array}\right)
$$

The corresponding vector field $\nabla_{H}$ is the Heisenberg gradient on the Heisenberggroup $\mathbb{H}^{n}$.

This is the simplest case of a more general setting: the Carnot groups. More details are given in Section 3.3. 
Example 2.4. (Heisenberg-Greiner operator) Let $\xi=(x, y, t) \in \mathbb{R}^{n} \times \mathbb{R}^{n} \times \mathbb{R}$, $r:=|(x, y)|, \gamma \geq 1$ and let $\mu$ be defined as

$$
\left(\begin{array}{ccc}
I_{n} & 0 & 2 \gamma y r^{2 \gamma-2} \\
0 & I_{n} & -2 \gamma x r^{2 \gamma-2}
\end{array}\right)
$$

The corresponding vector fields are $X_{i}=\frac{\partial}{\partial x_{i}}+2 \gamma y_{i} r^{2 \gamma-2} \frac{\partial}{\partial t}, Y_{i}=\frac{\partial}{\partial y_{i}}-2 \gamma x_{i} r^{2 \gamma-2} \frac{\partial}{\partial t}$ for $i=1, \ldots, n$.

For $\gamma=1 L$ is the sub-Laplacian $\Delta_{H}$ on the Heisenberg-group $\mathbb{H}^{n}$. If $\gamma=$ $2,3, \ldots, L$ is a Greiner operator (see [33]).

Let $A$ be an open subset of $\mathbb{R}^{N}$ with Lipschitz boundary $\partial A$ and let $\hat{h} \in$ $\mathscr{C}^{1}\left(\bar{A}, \mathbb{R}^{l}\right)$ be a vector field. By the divergence theorem we have

$$
\int_{A} \operatorname{div}_{L} \hat{h} d \xi=\int_{A} \operatorname{div}\left(\mu^{T} \hat{h}\right) d \xi=\int_{\partial A} \hat{h} \cdot \mu \nu d \Sigma=\int_{\partial A} \hat{h} \cdot \nu_{L} d \Sigma,
$$

where $v_{L}:=\mu \nu$, and $\nu$ denotes the exterior normal at point $\xi \in \partial A$. If $\hat{h}$ has the form $\hat{h}=f h$ with $f \in \mathscr{C}^{1}(\bar{A})$ and $h \in \mathscr{C}^{1}\left(\bar{A}, \mathbb{R}^{l}\right)$, then

$$
\int_{A} f \operatorname{div}_{L} h d \xi+\int_{A} \nabla_{L} f \cdot h d \xi=\int_{\partial A} f h \cdot v_{L} d \Sigma .
$$

Moreover, if $h=\nabla_{L} u$ with $u \in \mathscr{C}^{2}(\bar{A})$, then (2.4) yields the Gauss-Green formula

$$
\int_{A} f L u d \xi+\int_{A} \nabla_{L} f \cdot \nabla_{L} u d \xi=\int_{\partial A} f \nabla_{L} u \cdot v_{L} d \Sigma .
$$

Let $g \in \mathscr{C}^{1}(\mathbb{R})$ be such that $g(0)=0$ and let $\Omega \subset \mathbb{R}^{N}$ be open. For every vector field $h \in \mathscr{C}^{1}\left(\bar{A}, \mathbb{R}^{l}\right)$ and any compactly supported function $u \in \mathscr{C}_{0}^{1}(\Omega)$, choosing $f:=g(u)$ in (2.4), we obtain

$$
\int_{\Omega} g(u) \operatorname{div}_{L} h d \xi=-\int_{\Omega} g^{\prime}(u) \nabla_{L} u \cdot h d \xi .
$$

Let $h \in L_{\text {loc }}^{1}\left(\Omega, \mathbb{R}^{l}\right)$ be a vector field. As usual, we define the distribution $\operatorname{div}_{L} h$ using the formula (2.5) with $g(s)=s$. If in (2.5) we chose $g(t)=|t|^{p}$ with $p>1$, then for every $u \in \varnothing_{0}^{1}(\Omega)$ we have

$$
\int_{\Omega}|u|^{p} \operatorname{div}_{L} h d \xi=-p \int_{\Omega}|u|^{p-2} u \nabla_{L} u \cdot h d \xi .
$$

Let $h \in L_{\text {loc }}^{1}\left(\Omega, \mathbb{R}^{l}\right)$ be a vector field and let $A \in L_{\text {loc }}^{1}(\Omega)$ be a function. In what follows we write $A \leq \operatorname{div}_{L} h$ meaning that the inequality holds in distributional sense, that is for every $\phi \in \mathscr{C}_{0}^{1}(\Omega)$ such that $\phi \geq 0$, we have

$$
\int_{\Omega} \phi A d \xi \leq \int_{\Omega} \phi \operatorname{div}_{L} h d \xi=-\int_{\Omega} \nabla_{L} \phi \cdot h d \xi .
$$

Identities (2.5) and (2.6) play an important role in the proof of the following Hardytype inequalities and the Poincaré inequality too. 
Theorem 2.5. Let $p>1$. Let $h \in L_{\text {loc }}^{1}\left(\Omega, \mathbb{R}^{l}\right)$ be a vector field and let $A_{h} \in$ $L_{\mathrm{loc}}^{1}(\Omega)$ be a nonnegative function such that $A_{h} \leq \operatorname{div}_{L} h$ and $|h|^{p} A_{h}^{1-p} \in L_{\mathrm{loc}}^{1}(\Omega)$. Then for every $u \in \mathscr{C}_{0}^{1}(\Omega)$, we have

$$
\int_{\Omega}|u|^{p} A_{h} d \xi \leq p^{p} \int_{\Omega} \frac{|h|^{p}}{A_{h}^{(p-1)}}\left|\nabla_{L} u\right|^{p} d \xi .
$$

Proof. We note that the right hand side of (2.7) is finite since $u \in \mathscr{C}_{0}^{1}(\Omega)$. Using the identity (2.6) and Hölder inequality we obtain

$$
\begin{aligned}
\int_{\Omega}|u|^{p} A_{h} d \xi & \leq \int_{\Omega}|u|^{p} \operatorname{div}_{L} h d \xi \leq p \int_{\Omega}|u|^{p-1}|h|\left|\nabla_{L} u\right| d \xi \\
& =p \int_{\Omega}|u|^{p-1} A_{h}^{(p-1) / p} \frac{|h|}{A_{h}^{(p-1) / p}}\left|\nabla_{L} u\right| d \xi \\
& \leq p\left(\int_{\Omega}|u|^{p} A_{h} d \xi\right)^{(p-1) / p}\left(\int_{\Omega} \frac{|h|^{p}}{A_{h}^{p-1}}\left|\nabla_{L} u\right|^{p} d \xi\right)^{1 / p} .
\end{aligned}
$$

This completes the proof.

Specializing the vector field $h$ and the function $A_{h}$, we shall deduce from (2.7) some concrete inequalities of Hardy-type.

Remark 2.6. Setting $A_{h}=\operatorname{div}_{L} h$ in (2.7), we have

$$
\int_{\Omega}|u|^{p} \operatorname{div}_{L} h d \xi \leq p^{p} \int_{\Omega} \frac{|h|^{p}}{\left|\operatorname{div}_{L} h\right|^{(p-1)}}\left|\nabla_{L} u\right|^{p} d \xi .
$$

Acting as Davies and Hinz in [24], the choice $h:=\nabla_{L} V$ with $V$ such that $L V>0$, yields

$$
\int_{\Omega}|u|^{p}|L V| d \xi \leq p^{p} \int_{\Omega} \frac{\left|\nabla_{L} V\right|^{p}}{|L V|^{(p-1)}}\left|\nabla_{L} u\right|^{p} d \xi
$$

In order to state a Hardy inequality, now the problem is to find a suitable function $V$. In the Euclidean setting for $1<p<N$, choosing $V(\xi)=|\xi|^{2-p}$ if $1<p<2$, $V(\xi)=\ln |\xi|$ if $p=2$ and $V(\xi)=-|\xi|^{2-p}$ if $2<p<N$, we obtain the Hardy inequality (1.1) with $w(\xi)=|\xi|$.

Another strategy is to chose the vector field $h$ as $h=\left|\nabla_{L} V\right|^{p-2} \nabla_{L} V$ with $V$ such that $L_{p} V>0$. Thus, we have

$$
\int_{\Omega}|u|^{p}\left|L_{p} V\right| d \xi \leq p^{p} \int_{\Omega} \frac{\left|\nabla_{L} V\right|^{p(p-1)}}{\left|L_{p} V\right|^{(p-1)}}\left|\nabla_{L} u\right|^{p} d \xi .
$$


Hence, in the Euclidean setting for $1<p<N$, choosing $V(\xi)=\ln |\xi|$ we reobtain the inequality (1.1) with $w(\xi)=|\xi|$.

In order to obtain the classical Hardy inequalities in Euclidean setting, these strategies are equivalent. This equivalence is basically due to the fact that $|\nabla| \xi||=$ 1 for $\xi \neq 0$. The latter approach is slightly more simple: the choice of $V$ is independent of $p$. Moreover, it turned out to be more fruitful in the Heisenberg-group and in the Grushin plane settings (see $[19,18]$ ) as well as in our more general framework.

Let $d: \Omega \rightarrow \mathbb{R}$ be a nonnegative non constant measurable function. In order to state Hardy inequalities involving the weight $d$, the basic assumption we made on $d$ is that, for $\alpha \neq 0, d^{\alpha}$ is a one side weak solution of $-L_{p}(u)=0$, that is $d^{\alpha}$ is super- $L_{p}$-harmonic or sub- $L_{p}$-harmonic in weak sense. Namely, let $\alpha, \beta \in \mathbb{R}$, $\alpha \neq 0$, requiring

$$
d^{(\alpha-1)(p-1)}\left|\nabla_{L} d\right|^{p-1} \in L_{\mathrm{loc}}^{1}(\Omega)
$$

we assume that

$$
-L_{p}\left(d^{\alpha}\right) \geq 0 \quad \text { on } \Omega \quad[\text { resp. } \leq 0]
$$

in weak sense, that is for every nonnegative $\phi \in \mathscr{C}_{0}^{1}(\Omega)$ we have

$$
\begin{aligned}
\int_{\Omega}\left|\nabla_{L} d^{\alpha}\right|^{p-2} \nabla_{L} d^{\alpha} \cdot \nabla_{L} \phi & =\alpha|\alpha|^{p-2} \int_{\Omega} d^{(\alpha-1)(p-1)}\left|\nabla_{L} d\right|^{p-2} \nabla_{L} d \cdot \nabla_{L} \phi \\
& \geq 0[\text { resp. } \leq 0]
\end{aligned}
$$

and

$$
\alpha[(\alpha-1)(p-1)-\beta-1]>0, \quad[\text { resp. }<0] .
$$

Gluing together the above conditions, we assume that

$$
-L_{p}\left(c d^{\alpha}\right) \geq 0 \quad \text { on } \Omega
$$

in weak sense, where $c:=\alpha[(\alpha-1)(p-1)-\beta-1]$

Theorem 2.7. Assume that (2.11) and (2.15) hold. Let $\beta \in \mathbb{R}$ be such that

$$
\begin{aligned}
d^{\beta}\left|\nabla_{L} d\right|^{p} & \in L_{\mathrm{loc}}^{1}(\Omega), \\
d^{\beta+p} & \in L_{\mathrm{loc}}^{1}(\Omega) .
\end{aligned}
$$

For every function $u \in \mathscr{C}_{0}^{1}(\Omega)$, we have

$$
\left(c_{\alpha, \beta, p}\right)^{p} \int_{\Omega}|u|^{p} d^{\beta}\left|\nabla_{L} d\right|^{p} d \xi \leq \int_{\Omega} d^{\beta+p}\left|\nabla_{L} u\right|^{p} d \xi,
$$

where $c_{\alpha, \beta, p}:=|(\alpha-1)(p-1)-\beta-1| / p$. 
In particular, if $-L_{p}\left(d^{\alpha}\right) \geq 0$, then for every function $u \in \mathscr{C}_{0}^{1}(\Omega)$ we have

$$
\left(\frac{|\alpha|(p-1)}{p}\right)^{p} \int_{\Omega} \frac{|u|^{p}}{d^{p}}\left|\nabla_{L} d\right|^{p} d \xi \leq \int_{\Omega}\left|\nabla_{L} u\right|^{p} d \xi
$$

provided $d^{-p}\left|\nabla_{L} d\right|^{p} \in L_{\text {loc }}^{1}(\Omega)$.

Remark 2.8. In most examples we shall deal with, the constant $c_{\alpha, \beta, p}^{p}$, yielded by applying Theorem 2.7, results to be sharp. We shall now indicate an argument that can be used to prove the sharpness of the constant $c_{\alpha, \beta, p}^{p}$ involved in the inequality of Theorem 2.7. Let $c_{b}(\Omega)$ be the best constant in (2.18). It is clear that $c_{b}(\Omega) \geq$ $c_{\alpha, \beta, p}^{p}$. We shall assume that the hypotheses of Theorem 2.7 are satisfied and that there exists $s>0$ such that $\Omega^{s}:=d^{-1}(]-\infty, s[)$ and $\Omega_{s}:=d^{-1}(] s,+\infty[)$ are not empty open subsets of $\Omega$ with piecewise regular boundaries.

We assume that there exists $\epsilon_{0}>0$ such that for every $\left.\epsilon \in\right] 0, \epsilon_{0}[$ there hold

$$
0<\int_{d<s} d^{c(\epsilon) p+\beta}\left|\nabla_{L} d\right|^{p}<+\infty, \quad 0<\int_{d>s} d^{-c(\epsilon) p+\beta}\left|\nabla_{L} d\right|^{p}<+\infty,
$$

where

$$
c(\epsilon):=\frac{|(\alpha-1)(p-1)-\beta-1|+\epsilon}{p}=c_{\alpha, \beta, p}+\frac{\epsilon}{p} .
$$

By rescaling argument, we can assume that $s=1$. Let $\epsilon \in] 0, \epsilon_{0}[$ and let $v: \Omega \rightarrow \mathbb{R}$ be defined as

$$
v(\xi):= \begin{cases}d^{c(\epsilon)}(\xi) & \text { if } d(\xi) \leq 1, \\ d^{-c(\epsilon / 2)}(\xi) & \text { if } d(\xi)>1 .\end{cases}
$$

By hypothesis, $\int_{\Omega} v^{p} d^{\beta}\left|\nabla_{L} d\right|^{p}$ is finite. Thus, we have

$$
\begin{aligned}
c(\epsilon)^{p} \int_{\Omega} v^{p} d^{\beta}\left|\nabla_{L} d\right|^{p}= & c(\epsilon)^{p} \int_{d<1} d^{\beta+p} d^{(c(\epsilon)-1) p}\left|\nabla_{L} d\right|^{p} \\
& +c(\epsilon)^{p} \int_{d>1} d^{\beta+p} d^{(-c(\epsilon / 2)-1) p}\left|\nabla_{L} d\right|^{p} \\
= & \int_{d<1} d^{\beta+p}\left|\nabla_{L} v\right|^{p}+\left(\frac{c(\epsilon)}{c(\epsilon / 2)}\right)^{p} \int_{d>1} d^{\beta+p}\left|\nabla_{L} v\right|^{p} \\
= & \int_{\Omega} d^{\beta+p}\left|\nabla_{L} v\right|^{p}+\left(\frac{c(\epsilon)^{p}}{c(\epsilon / 2)^{p}}-1\right) \int_{d>1} d^{\beta+p}\left|\nabla_{L} v\right|^{p} .
\end{aligned}
$$

Observing that $c(\epsilon)>c(\epsilon / 2)$, we get

$$
c(\epsilon)^{p} \int_{\Omega} v^{p} d^{\beta}\left|\nabla_{L} d\right|^{p}>\int_{\Omega} d^{\beta+p}\left|\nabla_{L} v\right|^{p},
$$

the converse of the Hardy inequality. 
Now we assume that the Hardy inequality (2.18) holds for the function $v$ defined in (2.22). From (2.23) we deduce $c(\epsilon)^{p}>c_{b}(\Omega)$. Letting $\epsilon \rightarrow 0$, we get $c_{\alpha, \beta, p}^{p} \geq c_{b}(\Omega)$ and hence the claim.

The question of the existence of functions that realize the best constant arises. In such a general framework a unique answer cannot be given. Indeed, even in the Euclidean setting several cases occur. Let $p=2$, let $d_{1}(\cdot):=|\cdot|$ be the Euclidean distance from the origin, and let $d_{2}(\cdot):=\operatorname{dist}(\cdot, \partial \Omega)$ be the distance from the boundary of a given domain $\Omega$. If $\Omega \subset \mathbb{R}^{N}(N \geq 3)$ is a ball centered at the origin, then the best constants in the Hardy inequality (2.19) related to $d_{1}$ and $d_{2}$ are not achieved. On the other hand, there exist smooth bounded domains $\Omega$ such that the best constant in the inequality related to $d_{1}$ is not achieved and the best constant in the inequality related to $d_{2}$ is achieved (see $[40,41]$ ). Anyway, some steps in this direction can be done even in our general framework. For the sake of simplicity, we shall focus our attention on the inequality (2.19).

Therefore, under the same hypotheses of Theorem 2.7 we assume that $-L_{p}\left(d^{\alpha}\right) \geq 0$ on $\Omega$ in weak sense, that $\left(\int_{\Omega}\left|\nabla_{L} u\right|^{p} d \xi\right)^{1 / p}$ is a norm and that $D_{L}^{1, p}(\Omega)$, the closure of $\mathscr{C}_{0}^{\infty}(\Omega)$ in that norm, is well defined. We denote by $c_{b}(\Omega)$ the best constant in (2.19), namely

$$
c_{b}(\Omega):=\inf _{u \in D_{L}^{1, p}, u \neq 0} \frac{\int_{\Omega}\left|\nabla_{L} u\right|^{p} d \xi}{\int_{\Omega}|u|^{p} d^{-p}\left|\nabla_{L} d\right|^{p} d \xi} .
$$

Theorem 2.9. Under the above hypotheses we have:

1. If $d^{\alpha \frac{p-1}{p}} \in D_{L}^{1, p}(\Omega)$, then $c_{b}(\Omega)=\left(|\alpha| \frac{p-1}{p}\right)^{p}$ and $d^{\alpha \frac{p-1}{p}}$ is a minimizer.

2. If $d^{\alpha \frac{p-1}{p}} \notin D_{L}^{1, p}(\Omega), p \geq 2,\left|\nabla_{L} d\right| \neq 0$ a.e. and $c_{b}(\Omega)=\left(|\alpha| \frac{p-1}{p}\right)^{p}$ then the best constant $c_{b}(\Omega)$ is not achieved.

Remark 2.10. In all the examples we shall deal with in the last section, it is possible to apply Theorem 2.9 and, hence, for $p \geq 2$ the best constants mentioned in all the theorems of Section 3 are not achieved.

Remark 2.11. Let us to consider the special case of $\nabla_{L}=\nabla$, the usual Euclidean gradient, $d$ is the Euclidean distance from a given regular surface $K$ of codimension $k(1 \leq k \leq N), \alpha=\frac{p-k}{p-1}$ and $\beta=-p$. In this case, replacing $\Omega$ with $\Omega \backslash K$, Theorem 2.7 assures that the inequality

$$
\left(\frac{|p-k|}{p}\right)^{p} \int_{\Omega} \frac{|u|^{p}}{d^{p}} d \xi \leq \int_{\Omega}|\nabla u|^{p} d \xi
$$

holds for every $u \in \mathscr{C}_{0}^{1}(\Omega \backslash K)$ provided $-\Delta_{p}\left(d^{\alpha}\right) \geq 0$ on $\Omega \backslash K$.

This particular case of Theorem 2.7 is contained in $[4,5]$, where the authors also study the remainder terms for inequality (2.25).

The reader interested in the study of Hardy inequalities with remainder terms can refer to $[4,5,10,11,31]$ and the references therein for the Euclidean case and to [19] for the case $\nabla_{L}=\nabla_{H}$, the Heisenberg gradient on the Heisenberg-group. 
Proof of Theorem 2.7. We prove the thesis in the case $-L_{p}\left(d^{\alpha}\right) \geq 0$ and $c:=$ $\alpha[(\alpha-1)(p-1)-\beta-1]>0$. The alternative case is similar.

Let $\varphi \in \mathscr{C}_{0}^{1}(\Omega)$ be a nonnegative function. Choosing in $(2.13) \phi:=d^{\beta+1-(\alpha-1)(p-1)} \varphi$, we have

$$
\begin{aligned}
0 \leq & \alpha \int_{\Omega} d^{\beta+1}\left|\nabla_{L} d\right|^{p-2} \nabla_{L} d \cdot \nabla_{L} \varphi \\
& -\alpha[(\alpha-1)(p-1)-\beta-1] \int_{\Omega} d^{\beta}\left|\nabla_{L} d\right|^{p} \varphi .
\end{aligned}
$$

Using Hölder inequality and hypotheses (2.16) and (2.17), it is immediate to check that the above integrals are finite.

Let $h$ be the vector field defined by $h:=-\alpha d^{\beta+1}\left|\nabla_{L} d\right|^{p-2} \nabla_{L} d$ and let $A_{h}$ be the function defined as $A_{h}:=\alpha[(\alpha-1)(p-1)-\beta-1] d^{\beta}\left|\nabla_{L} d\right|^{p}$. Thus, from (2.26) and the fact that $c>0$, we obtain $\operatorname{div}_{L} h \geq A_{h} \geq 0$. Now we are in the position to apply Theorem 2.5 and this concludes the proof.

Proof of Theorem 2.9. 1) From (2.19), we have $c_{b}(\Omega) \geq\left(|\alpha| \frac{p-1}{p}\right)^{p}$. It is immediate to check that $u:=d^{\alpha \frac{p-1}{p}}$ realizes the infimum in (2.24).

2) Let $u \in \mathscr{C}_{0}^{\infty}(\Omega)$. We define the functional $I$ as

$$
I(u):=\int_{\Omega}\left|\nabla_{L} u\right|^{p} d \xi-\left(\frac{|\alpha|(p-1)}{p}\right)^{p} \int_{\Omega} \frac{|u|^{p}}{d^{p}}\left|\nabla_{L} d\right|^{p} d \xi .
$$

The functional $I$ is non negative, and the best constant will be achieved, if and only if, $I(u)=0$ for some $u \in D_{L}^{1, p}(\Omega)$.

Let $v$ be the new variable $v:=d^{-\gamma} u$ with $\gamma:=\alpha \frac{p-1}{p}$. By computation we have

$$
\left|\nabla_{L} u\right|^{2}=|\gamma|^{2} v^{2} d^{2 \gamma-2}\left|\nabla_{L} d\right|^{2}+d^{2 \gamma}\left|\nabla_{L} v\right|^{2}+2 \gamma v d^{2 \gamma-1}\left(\nabla_{L} d \cdot \nabla_{L} v\right) .
$$

(If $d$ is not smooth enough, by standard argument one can consider $d_{\epsilon}$ a regularization of $d$ and after the computation taking the limit as $\epsilon \rightarrow 0$ ).

We remind that the inequality

$$
(\xi-\eta)^{s} \geq \xi^{s}-s \eta \xi^{s-1}
$$

holds for every $\xi, \eta, s \in \mathbb{R}$ with $\xi>0, \xi>\eta$ and $s \geq 1$ (see [31]). Applying (2.28) and (2.27) with $s=p / 2, \xi=|\gamma|^{2} v^{2} d^{2 \gamma-2}\left|\nabla_{L} d\right|^{2}$ and $\eta=-2 \gamma v d^{2 \gamma-1}\left(\nabla_{L} d\right.$. $\left.\nabla_{L} v\right)-d^{2 \gamma}\left|\nabla_{L} v\right|^{2}$, we have

$$
\begin{aligned}
\left|\nabla_{L} u\right|^{p} \geq & |\gamma|^{p} v^{p} d^{\gamma p} d^{-p}\left|\nabla_{L} d\right|^{p} \\
& +p|\gamma|^{p-2} \gamma|v|^{p-2} v d^{(\alpha-1)(p-1)}\left|\nabla_{L} d\right|^{p-2}\left(\nabla_{L} d \cdot \nabla_{L} v\right) \\
& +\frac{p}{2}|\gamma|^{p-2}|v|^{p-2} d^{(\alpha-1)(p-1)+1}\left|\nabla_{L} d\right|^{p-2}\left|\nabla_{L} v\right|^{2} .
\end{aligned}
$$


Taking into account that $u:=d^{\gamma} v$ we have

$$
I(u) \geq I_{1}(v)+I_{2}(v)
$$

where

$$
\begin{aligned}
& I_{1}(v):=\int_{\Omega} p|\gamma|^{p-2} \gamma|v|^{p-2} v d^{(\alpha-1)(p-1)}\left|\nabla_{L} d\right|^{p-2}\left(\nabla_{L} d \cdot \nabla_{L} v\right) d \xi \\
& I_{2}(v):=\frac{p}{2}|\gamma|^{p-2} \int_{\Omega}|v|^{p-2} d^{(\alpha-1)(p-1)+1}\left|\nabla_{L} d\right|^{p-2}\left|\nabla_{L} v\right|^{2} d \xi
\end{aligned}
$$

Re-arranging the expression in $I_{1}$ and integrating by parts we obtain

$$
\begin{aligned}
I_{1}(v)= & \left(\frac{p-1}{p}\right)^{p-1} \int_{\Omega}\left(\nabla_{L}|v|^{p} \cdot\left|\nabla_{L} d^{\alpha}\right|^{p-2} \nabla_{L} d^{\alpha}\right) d \xi \\
= & \left(\frac{p-1}{p}\right)^{p-1} \int_{\partial \Omega}|v|^{p}\left|\nabla_{L} d^{\alpha}\right|^{p-2}\left(\nabla_{L} d^{\alpha} \cdot v_{L}\right) d \Sigma \\
& +\left(\frac{p-1}{p}\right)^{p-1} \int_{\Omega}|v|^{p}\left(-L_{p}\left(d^{\alpha}\right)\right) d \xi \geq 0,
\end{aligned}
$$

where we have used the fact that $v \in \mathscr{C}_{0}^{\infty}(\Omega)$ and the hypothesis $-L_{p}\left(d^{\alpha}\right) \geq 0$. On the other hand we can rewrite $I_{2}$ as

$$
I_{2}(v)=\left.\left.\frac{2}{p}|\gamma|^{p-2} \int_{\Omega} d^{(\alpha-1)(p-1)+1}\left|\nabla_{L} d\right|^{p-2}\left|\nabla_{L}\right| v\right|^{\frac{p}{2}}\right|^{2} d \xi .
$$

Thus, we conclude that for any $u \in D_{L}^{1, p}(\Omega)$

$$
I(u) \geq\left.\left.\frac{2}{p}|\gamma|^{p-2} \int_{\Omega} d^{(\alpha-1)(p-1)+1}\left|\nabla_{L} d\right|^{p-2}\left|\nabla_{L}\right| v\right|^{\frac{p}{2}}\right|^{2} d \xi,
$$

and this inequality implies the non existence of minimizers in $D_{L}^{1, p}(\Omega)$.

Specializing the function $d$, we shall deduce from Theorem (2.7) some concrete inequalities of Hardy-type. A first example is the following. We assume that there exists $m \in \mathbb{N}, 1 \leq m \leq l$ such that the matrix $\mu$ in (2.1) has the following form

$$
\mu:=\left(\begin{array}{cc}
I_{m} & \mu_{1} \\
0 & \mu_{2}
\end{array}\right)
$$

where $\mu_{1}$ and $\mu_{2}$ denote matrixes with $m \times(N-m)$ and $(l-m) \times(N-m)$ continuous entries respectively and $I_{m}$ stands for the identity matrix of order $m$. Notice that this case occurs in all the examples cited above. 
Set $\eta:=\left(\xi_{1}, \ldots, \xi_{m}\right), \tau:=\left(\xi_{m+1}, \ldots, \xi_{N}\right)$ and let $v_{p}$ be defined for $\eta \in$ $\mathbb{R}^{m} \backslash\{0\}$ as

$$
v_{p}(\eta):= \begin{cases}|\eta|^{\frac{p-m}{p-1}} & \text { if } p \neq m, \\ -\ln |\eta| & \text { if } p=m .\end{cases}
$$

The function $v_{p}$ is $p$-harmonic on $\mathbb{R}^{m} \backslash\{0\} \times \mathbb{R}^{N-m}$ for the Euclidean $p$-Laplacian acting on the $\eta$ variable $\Delta_{p, \eta}$ and hence also for the quasilinear operator $L_{p}$. Moreover, there exists a constant $l_{p} \neq 0$ such that

$$
-\Delta_{p, \eta} v_{p}=l_{p} \delta_{0} \quad \text { on } \mathbb{R}^{m}
$$

in weak sense, where $\delta_{0}$ is the Dirac distribution at $0 \in \mathbb{R}^{m}$ and $l_{p}>0$ if and only if $1<p \leq m$. These relations allow us to apply Theorem 2.7.

Theorem 2.12. Assume that $\mu$ has the form (2.29) and let $\beta \in \mathbb{R}$ be fixed.

1. Let $1<p<\infty$ and let $\Omega \subset \mathbb{R}^{N}$ be an open set. If $m+\beta<0$, then we also require that $\Omega \subset\left(\mathbb{R}^{m} \backslash\{0\}\right) \times \mathbb{R}^{N-m}$. Then for every $u \in \mathscr{C}_{0}^{1}(\Omega)$, we have

$$
b_{\beta}^{p} \int_{\Omega}|u(\eta, \tau)|^{p}|\eta|^{\beta} d \eta d \tau \leq \int_{\Omega}\left|\nabla_{L} u(\eta, \tau)\right|^{p}|\eta|^{p+\beta} d \eta d \tau,
$$

where $b_{\beta}:=\frac{|m+\beta|}{p}$.

In particular, for every $u \in \mathscr{C}_{0}^{1}(\Omega)$, we obtain

$$
\begin{aligned}
\left(\frac{|m-p|}{p}\right)^{p} \int_{\Omega} \frac{|u(\xi)|^{p}}{|\xi|^{p}} d \xi & \leq\left(\frac{|m-p|}{p}\right)^{p} \int_{\Omega} \frac{|u(\eta, \tau)|^{p}}{|\eta|^{p}} d \eta d \tau \\
& \leq \int_{\Omega}\left|\nabla_{L} u(\xi)\right|^{p} d \xi
\end{aligned}
$$

2. Let $p=m>1$. Let $R>0$ and set $\Omega:=\left\{\xi=(\eta, \tau) \in \mathbb{R}^{m} \times \mathbb{R}^{N-m},|\eta|<R\right\}$. If $\beta<-1$, then for every $u \in \mathscr{C}_{0}^{1}(\Omega)$, we have

$$
\tilde{b}_{\beta}^{p} \int_{\Omega} \frac{|u(\eta, \tau)|^{p}}{|\eta|^{p}}\left(\ln \frac{R}{|\eta|}\right)^{\beta} d \eta d \tau \leq \int_{\Omega}\left|\nabla_{L} u(\eta, \tau)\right|^{p}\left(\ln \frac{R}{|\eta|}\right)^{p+\beta} d \eta d \tau,
$$

where $\tilde{b}_{\beta}:=\frac{|\beta+1|}{p}$.

In particular, for every $u \in \mathscr{C}_{0}^{1}(\Omega)$, we obtain

$$
\left(\frac{p-1}{p}\right)^{p} \int_{\Omega} \frac{|u(\eta, \tau)|^{p}}{(|\eta| \ln (R /|\eta|))^{p}} d \eta d \tau \leq \int_{\Omega}\left|\nabla_{L} u(\xi)\right|^{p} d \xi .
$$

Remark 2.13. It is easy to check that the inequality (2.33) holds also for $\beta>-1$ provided the set $\Omega$ is replaced by $\Omega:=\left\{(\eta, \tau) \in \mathbb{R}^{m} \times \mathbb{R}^{N-m}, 0<|\eta|<R\right\}$. 
Proof. Let $1<p<m$. We claim that the function $v_{p}$ is super- $L_{p}$-harmonic on $\mathbb{R}^{N}$. Indeed, let $\phi \in \mathscr{C}_{0}^{1}\left(\mathbb{R}^{N}\right)$ be a non negative function. Observing that $\left|\nabla_{L} v_{p}\right|=\left|\nabla_{\eta} v_{p}\right|$, we have

$$
\begin{aligned}
\int_{\mathbb{R}^{N}}-L_{p} v_{p} \phi d \xi & =\int_{\mathbb{R}^{N-m}} d \tau \int_{\mathbb{R}^{m}} d \eta\left(-\Delta_{p, \eta} v_{p}\right) \phi \\
& =l_{p} \int_{\mathbb{R}^{N-m}} \phi(0, \tau) d \tau \geq 0
\end{aligned}
$$

Analogously, one can prove that $v_{p}$ is super- $L_{p}$-harmonic when $p=m$ and sub$L_{p}$-harmonic when $p>m$.

First we consider the case $p \neq m$. We choose $d^{\alpha}=v_{p}$ with $d(\xi)=d(\eta, \tau)=$ $|\eta|$ and $\alpha=\frac{p-m}{p-1}$. Observing that $\left|\nabla_{L}\right| \eta||=\left|\nabla_{\eta}\right| \eta||=1$ a.e. and that the integrability conditions (2.11),(2.16), (2.17) are satisfied, applying Theorem 2.7 we get (2.31).

Let $p=m>1$. The choices $d(\eta, \tau)=\ln \frac{R}{|\eta|}$ and $\alpha=1$ in Theorem 2.7 yield the inequality (2.33).

Finally, we prove the missing inequality (2.31) when $p=m$. We consider the case $m+\beta>0$. The case $m+\beta<0$ is analogous and the case $m+\beta=0$ is trivial. Let $\sigma>0$ be such that $m+\beta-\sigma>0$. We chose $d(\xi)=|\eta|, \alpha=\frac{\sigma}{m-1}$. In this case it easy to check that $d^{\alpha}$ is sub- $L_{m}$-harmonic on $\mathbb{R}^{N}$, that is

$$
-L_{m}\left(d^{\alpha}\right)=-\operatorname{div}_{L}\left(\left(\frac{\sigma}{m-1}\right)^{m-1}|\eta|^{\sigma-m+1} \nabla_{\eta}|\eta|\right)=-\frac{\sigma^{m}}{(m-1)^{m-1}} \frac{1}{|\eta|^{m-\sigma}} \leq 0 .
$$

The constant $c$ in (2.15) is $c=\frac{\sigma}{m-1} \frac{\sigma-m-\beta}{m}<0$. Hence, we are in the position to apply Theorem 2.7; thus, we derive the inequality

$$
\left(\frac{m+\beta-\sigma}{p}\right)^{p} \int_{\Omega}|u|^{p}|\eta|^{\beta} d \xi \leq \int_{\Omega}\left|\nabla_{L} u\right|^{p}|\eta|^{p+\beta} d \xi .
$$

Letting $\sigma \rightarrow 0$, we get the claim.

Remark 2.14. In the case $\mu=I_{N}$, the vector field $\nabla_{L}$ is the usual gradient $\nabla$. For $m<N$, inequalities of type (2.31) are already present in [43] and in [44]. Secchi, Smets and Willem in [48] prove that the constant $b_{\beta}^{p}$ is optimal when $m+\beta>0$ and $\Omega=\mathbb{R}^{N}$ (see next section for further generalization in this direction).

An immediate consequence of Theorem 2.12 is a Poincare inequality for the vector field $\nabla_{L}$. The claim easily follows from inequality (2.31) with $\beta=0$.

Theorem 2.15. Let $\Omega$ be an open subset of $\mathbb{R}^{N}$ bounded in $\xi_{1}$ direction, that is, there exists $M>0$ such that for every $\xi \in \Omega$ it results $\left|\xi_{1}\right| \leq M$. Assume that the matrix $\mu$ has the form (2.29). 
Then, for every $u \in \varnothing_{0}^{1}(\Omega)$, we have

$$
c^{p} \int_{\Omega}|u|^{p} d \xi \leq \int_{\Omega}\left|\nabla_{L} u\right|^{p} d \xi
$$

with $c:=\frac{1}{p M}$.

In [21] the authors, in order to study the inequality $-L u \geq f$, make the same assumptions on the operator $L$. Namely, for a fixed $\eta \in \mathbb{R}^{N}$ they say that $\left(\mathbf{H}_{\eta}\right)$ is satisfied if there exist a real number $Q=Q(\eta)>2$ and a nonnegative continuous function $d_{\eta}: \mathbb{R}^{N} \rightarrow \mathbb{R}_{+}$such that the following four properties hold

1. $d_{\eta}(\xi)=0$ if and only if $\xi=\eta$.

2. $d_{\eta} \in \complement^{2}\left(\mathbb{R}^{N} \backslash\{\eta\}\right)$.

3. The fundamental solution of $-L$ on $\mathbb{R}^{N}$ at $\eta$ is given by $\Gamma_{\eta}=d_{\eta}^{2-Q}$. That is, the functions $d_{\eta}^{2-Q}$ and $d_{\eta}^{1-Q}$ belong to $L_{\text {loc }}^{1}\left(\mathbb{R}^{N}\right)$ and for any $\phi \in \varnothing_{0}^{2}\left(\mathbb{R}^{N}\right)$ we have

$$
\int_{\mathbb{R}^{N}}(-L \phi)(\xi) \Gamma_{\eta}(\xi) d \xi=\phi(\eta) .
$$

4. For any $i, j=1, \ldots, l$ the functions $X_{i} d_{\eta}, X_{j}\left(d_{\eta} X_{i} d_{\eta}\right)$ are bounded and $\left|\nabla_{L} d_{\eta}\right|^{2} \neq 0$ almost everywhere on $\mathbb{R}^{N}$.

In this setting, it is immediate to check that the hypotheses of Theorem 2.7 are fulfilled and a Hardy inequality related to the operator $L$ holds.

Theorem 2.16. Let $\eta \in \mathbb{R}^{N}$ and assume that $\left(\mathbf{H}_{\eta}\right)$ is satisfied. Then for any $u \in$ $C_{0}^{1}\left(\mathbb{R}^{N}\right)$, we have

$$
\left(\frac{Q(\eta)-2}{2}\right)^{2} \int_{\mathbb{R}^{N}} \frac{u^{2}(\xi)}{d_{\eta}^{2}(\xi)}\left|\nabla_{L} d_{\eta}(\xi)\right|^{2} d \xi \leq \int_{\mathbb{R}^{N}}\left|\nabla_{L} u(\xi)\right|^{2} d \xi
$$

As particular case of Theorem 2.7, we obtain the following

Theorem 2.17. Let $g \in \complement^{2}(\Omega)$ be an $L_{p}$-harmonic function, that is $L_{p} g=0$ and let $v \in \mathscr{C}^{2}(\mathbb{R})$ be a concave function such that $v \circ g$ is positive on $\Omega$. For any $u \in \mathscr{C}_{0}^{1}(\Omega)$ we have

$$
\left(\frac{p-1}{p}\right)^{p} \int_{\Omega}|u(\xi)|^{p} \frac{\left|v^{\prime}(g(\xi))\right|^{p}}{v^{p}(g(\xi))}\left|\nabla_{L} g(\xi)\right|^{p} d \xi \leq \int_{\Omega}\left|\nabla_{L} u(\xi)\right|^{p} d \xi .
$$

The above result follows from Theorem 2.7 and the following worthwhile lemma 
Lemma 2.18. Let $p>1, \alpha \in \mathbb{R}, \alpha \neq 0$ and $g \in \mathscr{C}^{2}(\Omega)$ be a positive function such that $L_{p}\left(g^{\alpha}\right)=0$. Let $v \in \mathscr{C}^{2}(\mathbb{R})$ be such that $v^{\prime}(g(\xi)) \neq 0$ for $\xi \in \Omega$. Then setting $u(\xi):=v(g(\xi))$, we have

$$
L_{p} u=(p-1)\left|\nabla_{L} g\right|^{p}\left|v^{\prime}(g)\right|^{p-2}\left[v^{\prime \prime}+\frac{1-\alpha}{g} v^{\prime}\right] .
$$

Proof. We notice that for every $f \in \mathscr{C}^{1}(\Omega)$ and $h \in \mathscr{C}^{1}\left(\Omega, \mathbb{R}^{l}\right)$, we have $-\nabla_{L}^{*}(f h)=$ $\nabla_{L} f \cdot h-f \nabla_{L}^{*} h$. Let $\phi \in \mathscr{C}^{1}(\mathbb{R})$. Observing that $\nabla_{L}(\phi \circ g)=\phi^{\prime}(g) \nabla_{L} g$, we deduce

$$
\begin{aligned}
L_{p} \phi & =-\nabla_{L}^{*}\left(\left|\phi^{\prime}(g)\right|^{p-2}\left|\nabla_{L} g\right|^{p-2} \phi^{\prime}(g) \nabla_{L} g\right) \\
& =\left|\phi^{\prime}(g)\right|^{p-2}\left[(p-1) \phi^{\prime \prime}\left|\nabla_{L} g\right|^{p}+\phi^{\prime} L_{p} g\right] .
\end{aligned}
$$

Choosing $\phi(t)=t^{\alpha}$ in (2.36) and taking into account the $p$-harmonicity of $g^{\alpha}$ we obtain

$$
g L_{p} g=(p-1)(1-\alpha)\left|\nabla_{L} g\right|^{p},
$$

which substituted in (2.36), yields the claim.

Remark 2.19. Lemma 2.18 provides a generalization of the expression of the usual $p$-Laplacian for radial function.

As simple application of previous results is the following

Proposition 2.20. Let $\nabla_{L}$ be the usual gradient in $\mathbb{R}^{2}$, that is $\nabla_{L}:=\nabla=\left(\partial_{x}, \partial_{y}\right)^{T}$ and $\Omega:=]-\frac{\pi}{2}, \frac{\pi}{2}\left[\times \mathbb{R}\right.$. For every $u \in \mathscr{C}_{0}^{1}(\Omega)$ we have

$$
\frac{1}{4} \int_{\Omega} \frac{u^{2}(x, y)}{\cos ^{2} x} d x d y \leq \int_{\Omega}|\nabla u(x, y)|^{2} d x d y .
$$

Moreover the constant $1 / 4$ is optimal and it is not achieved in $D^{1,2}(\Omega)$.

Proof. The inequality (2.37) follows from Theorem 2.7 and the choice $d(x, y):=$ $e^{y} \cos x$.

Merging (2.37) and the inequality

$$
\cos x \leq \frac{\pi}{2}-|x|=\operatorname{dist}((x, y), \partial \Omega) \text { for }(x, y) \in \Omega,
$$

we have

$$
\frac{1}{4} \int_{\Omega} \frac{u^{2}(x, y)}{\operatorname{dist}^{2}((x, y), \partial \Omega)} d x d y \leq \int_{\Omega}|\nabla u(x, y)|^{2} d x d y .
$$

The fact that $1 / 4$ is the best constant in (2.38) (see [41]), implies the optimality of $1 / 4$ in (2.37). Hence applying Theorem 2.9 we conclude the proof.

Remark 2.21. Finally, we notice that the result stated in our main Theorem 2.7 can be reformulated also for non compact Riemannian manifold. This allow us to reobtain the Hardy inequalities present in [16] as well as their many generalizations with weaker hypotheses. 


\section{Hardy inequalities for some subelliptic operators}

In this section we shall apply the previous results to particular operators.

Let $1<p<\infty$. In the sequel for a given vector field $\nabla_{L}$ and open set $\Omega \subset \mathbb{R}^{N}$, we shall denote by $D_{L}^{1, p}(\Omega)$ the closure of $\mathscr{C}_{0}^{\infty}(\Omega)$ in the norm $\left(\int_{\Omega}\left|\nabla_{L} u\right|^{p} d \xi\right)^{1 / p}$. If $w \in L_{\mathrm{loc}}^{1}(\Omega)$ and $w>0$ a.e. on $\Omega, D_{L}^{1, p}(\Omega, w)$ denotes the closure of $\mathscr{C}_{0}^{\infty}(\Omega)$ in the norm $\left(\int_{\Omega}\left|\nabla_{L} u\right|^{p} w d \xi\right)^{1 / p}$.

\subsection{Baouendi-Grushin operator}

Let $\mathbb{R}^{N}$ be splitted in $\xi=(x, y) \in \mathbb{R}^{n} \times \mathbb{R}^{k}$. Let $\gamma \geq 0$ be a nonnegative real number and let $\mu$ be the matrix defined in (2.2). The corresponding vector field is $\nabla_{\gamma}=\left(\nabla_{x},|x|^{\gamma} \nabla_{y}\right)$ and the nonlinear operator $L_{p}$ is $L_{p} u=\operatorname{div}_{L}\left(\left|\nabla_{\gamma} u\right|^{p-2} \nabla_{\gamma} u\right)$. The linear operator $L=L_{2}$ is the so-called Baouendi-Grushin operator $L=\Delta_{\gamma}=$ $\Delta_{x}+|x|^{2 \gamma} \Delta_{y}$. Notice that if $k=0$ or $\gamma=0$, then $L$ and $L_{p}$ coincide respectively with the usual Laplacian operator and $p$-Laplacian operator.

Defining on $\mathbb{R}^{N}$ the dilation $\delta_{\lambda}$ as

$$
\delta_{\lambda}(x, y):=\left(\lambda x, \lambda^{1+\gamma} y\right)
$$

it is not difficult to check that $\nabla_{\gamma}$ is homogeneous of degree one with respect to the dilation: $\nabla_{\gamma}\left(\delta_{\lambda}\right)=\lambda \delta_{\lambda}\left(\nabla_{\gamma}\right)$.

Let $\llbracket \xi \rrbracket=\llbracket(x, y) \rrbracket$ be the following distance from the origin on $\mathbb{R}^{N}$ :

$$
\llbracket \xi \rrbracket=\llbracket(x, y) \rrbracket:=\left(\left(\sum_{i=1}^{d} x_{i}^{2}\right)^{1+\gamma}+(1+\gamma)^{2} \sum_{i=1}^{k} y_{i}^{2}\right)^{\frac{1}{2+2 \gamma}} .
$$

It is easy to see that $\mathbb{[} \cdot \rrbracket$ is homogeneous of degree one with respect to $\delta_{\lambda}$.

Let $Q:=n+(1+\gamma) k$ be the so called homogeneous dimension. If for $R>0$ we denote by $B_{R}$ the set

$$
B_{R}:=\left\{\xi \in \mathbb{R}^{N}: \llbracket \xi \rrbracket<R\right\},
$$

then we have

$$
\left|B_{R}\right|=\left|B_{1}\right| R^{Q} .
$$

Moreover, we have that $\llbracket \xi \rrbracket^{s} \in L_{\mathrm{loc}}^{1}\left(\mathbb{R}^{N}\right)$ if and only if $s>-Q$ and $\llbracket \xi \rrbracket^{s} \in$ $L^{1}\left(\mathbb{R}^{N} \backslash B_{1}\right)$ if and only if $s<-Q$.

The function $\llbracket \cdot \rrbracket$ is related to the fundamental solution at the origin of Grushin operator $L$ (see [20]). Namely, if $Q>2$ then the function $u_{2}:=\llbracket \xi \rrbracket^{2-Q}$ satisfies the relation

$$
-L u_{2}=l_{2} \delta_{0} \quad \text { on } \mathbb{R}^{N}
$$

in weak sense, where $\delta_{0}$ is the Dirac measure at 0 and $l_{2}$ is a positive constant. 
It is immediate to check that

$$
\left|\nabla_{\gamma} \llbracket \xi \rrbracket\right|=\frac{|x|^{\gamma}}{\llbracket \xi \rrbracket^{\gamma}} \leq 1 .
$$

Let $p>1$ and let $\Gamma_{p}$ be the function defined as

$$
\Gamma_{p}(\xi):=\left\{\begin{array}{l}
\llbracket \xi \rrbracket^{\frac{p-Q}{p-1}} \text { if } p \neq Q \\
-\ln \llbracket \xi \rrbracket \text { if } p=Q
\end{array} \quad \text { for } \xi \neq 0\right.
$$

A direct calculation gives that $\Gamma_{p}$ is $L_{p}$ harmonic on $\mathbb{R}^{N} \backslash\{0\}$, that is

$$
-L_{p} \Gamma_{p}=0 \quad \text { on } \mathbb{R}^{N} \backslash\{0\} .
$$

Moreover, with analogous computations of [2], it is possible to show that there exists a constant $l_{p} \neq 0$ such that

$$
-L_{p} \Gamma_{p}=l_{p} \delta_{0} \quad \text { on } \mathbb{R}^{N}
$$

in weak sense and $l_{p}>0$ if and only if $Q \geq p>1$ (see also [6]).

Theorem 3.1. Let $\beta \in \mathbb{R}$ be fixed.

1. Let $1<p<+\infty$ and let $\Omega \subset \mathbb{R}^{N}$ be an open set. If $\beta+Q<0$, then we also require that $0 \notin \Omega$. We have

$$
\begin{array}{r}
c_{\beta}^{p} \int_{\Omega}|u|^{p} \llbracket \xi \rrbracket^{\beta} \frac{|x|^{\gamma p}}{\llbracket \xi \rrbracket^{\gamma p}} d \xi \leq \int_{\Omega}\left|\nabla_{\gamma} u\right|^{p} \llbracket \xi \rrbracket^{p+\beta} d \xi \\
u \in D_{L}^{1, p}\left(\Omega, \llbracket \xi \rrbracket^{p+\beta}\right),
\end{array}
$$

where $c_{\beta}:=\frac{|Q+\beta|}{p}$. In particular, we obtain

$$
\left(\frac{|Q-p|}{p}\right)^{p} \int_{\Omega} \frac{|u|^{p}}{\llbracket \xi \rrbracket^{p}} \frac{|x|^{\gamma p}}{\llbracket \xi \rrbracket^{\gamma p}} d \xi \leq \int_{\Omega}\left|\nabla_{\gamma} u\right|^{p} d \xi \quad u \in D_{L}^{1, p}(\Omega) .
$$

Moreover, if $\Omega \cup\{0\}$ is a neighbourhood of the origin, then the constant $c_{\beta}^{p}$ is sharp.

2. Let $p=Q>1$. Let $R>0$ and set $\Omega:=\left\{\xi \in \mathbb{R}^{N} \mid \llbracket \xi \rrbracket<R\right\}$. If $\beta<-1$, then we have

$$
\begin{aligned}
\tilde{c}_{\beta}^{p} \int_{\Omega} \frac{|u|^{p}}{\llbracket \xi \rrbracket^{p}}\left(\ln \frac{R}{\llbracket \xi \rrbracket}\right)^{\beta} \frac{|x|^{\gamma p}}{\llbracket \xi \rrbracket^{\gamma p}} d \xi \leq \int_{\Omega}\left|\nabla_{\gamma} u\right|^{p}\left(\ln \frac{R}{\llbracket \xi \rrbracket}\right)^{p+\beta} d \xi \\
u \in D_{L}^{1, p}\left(\Omega,(\ln (R / \llbracket \xi \rrbracket))^{p+\beta}\right),
\end{aligned}
$$


where $\tilde{c}_{\beta}:=\frac{|\beta+1|}{p}$. In particular, we obtain

$$
\begin{array}{r}
\left(\frac{p-1}{p}\right)^{p} \int_{\Omega} \frac{|u|^{p}}{(\llbracket \xi \rrbracket \ln (R / \llbracket \xi \rrbracket))^{p}} \frac{|x|^{\gamma p}}{\llbracket \xi \rrbracket^{\gamma p}} d \xi \leq \int_{\Omega}\left|\nabla_{\gamma} u\right|^{p} d \xi \\
u \in D_{L}^{1, p}(\Omega) .
\end{array}
$$

Moreover, the constant $\tilde{c}_{\beta}^{p}$ is sharp.

Remark 3.2. If $\gamma=0$, then the operator $\Delta_{\gamma}$ is the standard Laplacian operator acting on functions defined on $\mathbb{R}^{N}$ and (3.7) is the classical Hardy inequality (see (1.1) where $w(\xi)=|\xi|)$.

Remark 3.3. The above inequality (3.6) is already obtained in the case $1<p<Q$ by the author in [18].

Remark 3.4. The inequality (3.8) holds also for any $\beta>-1$ provided the set $\Omega$ is replaced by $\Omega:=\left\{\xi \in \mathbb{R}^{N} \mid 0<\llbracket \xi \rrbracket<R\right\}$.

Proof. We shall prove the inequalities for $u \in C_{0}^{1}(\Omega)$. The general case will follows by density argument.

The inequalities (3.6) for $p \neq Q$, and (3.8) follow from Theorem 2.7 choosing $d^{\alpha}=\Gamma_{p}$.

Now, we prove the missing inequality (3.6) when $p=Q$. We consider the case $Q+\beta>0$, the converse case is similar. Let $\sigma>0$ be such that $Q+\beta-\sigma>0$. We choose $d(\xi)=\llbracket \xi \rrbracket$ and $\alpha=\frac{\sigma}{Q-1}$. In this case it easy to check that $d^{\alpha}$ is sub- $L_{Q}$-harmonic on $\mathbb{R}^{N}$, that is

$$
-L_{Q}\left(d^{\alpha}\right)=-\operatorname{div}_{L}\left(\left(\frac{\sigma}{Q-1}\right)^{Q-1} \llbracket \xi \rrbracket^{\sigma} \frac{\left|\nabla_{L} \llbracket \xi \rrbracket\right|^{Q-2}}{\llbracket \xi \rrbracket^{Q-1}} \nabla_{L} \llbracket \xi \rrbracket\right) \leq 0
$$

in weak sense. Indeed, using (3.4) with $p=Q$, we deduce

$$
\begin{aligned}
-L_{Q}\left(d^{\alpha}\right) & =-\left(\frac{\sigma}{Q-1}\right)^{Q-1} \llbracket \xi \rrbracket^{\sigma} \operatorname{div}_{L}(\Gamma Q)-\sigma\left(\frac{\sigma}{Q-1}\right)^{Q-1} \frac{\left|\nabla_{L} \llbracket \xi \rrbracket\right|^{Q}}{\llbracket \xi \rrbracket^{Q-\sigma}} \\
& =\left(\frac{\sigma}{Q-1}\right)^{Q-1} \llbracket \xi \rrbracket^{\sigma} \frac{\delta_{0}}{l_{Q}}-\frac{\sigma}{(Q-1)^{Q-1}} \frac{\mid \nabla_{L} \llbracket \xi \rrbracket^{Q}}{\llbracket \xi \rrbracket^{Q-\sigma}} .
\end{aligned}
$$

Therefore, we get $-L_{Q}\left(d^{\alpha}\right)=-\frac{\sigma^{Q}}{(Q-1)^{Q-1}} \frac{\mid \nabla_{L} \llbracket \xi \|^{Q}}{\llbracket \xi \rrbracket^{Q-\sigma}} \leq 0$ in weak sense. The constant $c$ in (2.15) is $c=\frac{\sigma}{Q-1} \frac{\sigma-Q-\beta}{Q}<0$.

These choices yield the inequality

$$
\left(\frac{Q+\beta-\sigma}{Q}\right)^{p} \int_{\Omega}|u|^{p} \llbracket \xi \rrbracket^{\beta} \frac{|x|^{\gamma p}}{\llbracket \xi \rrbracket^{\gamma p}} d \xi \leq \int_{\Omega}\left|\nabla_{\gamma} u\right|^{p} \llbracket \xi \rrbracket^{p+\beta} d \xi .
$$

Letting $\sigma \rightarrow 0$ we get the thesis. 
It remains to show that the constants $c_{\beta}^{p}$ and $\tilde{c}_{\beta}^{p}$ appearing in (3.6) and in (3.8) are sharp. First we consider the case $\Omega=\mathbb{R}^{N} \backslash\{0\}$. To this end it suffices to show that we are in the position to apply the scheme outlined in Remark 2.8.

Indeed, let $d(\xi):=\llbracket \xi \rrbracket$ and for $\epsilon>0$ consider the constant $c(\epsilon)=\frac{Q+\beta}{p}+\frac{\epsilon}{p}$ defined in (2.21). We have

$$
c(\epsilon) p+\beta=|Q+\beta|+Q+\beta+Q-Q+\epsilon \geq-Q+\epsilon
$$

and

$$
-c(\epsilon) p+\beta=-|Q+\beta|+\beta+Q-Q-\epsilon \leq-Q-\epsilon .
$$

These inequalities imply the integrability conditions (2.20). Hence we obtain the sharpness of the constants.

In order to conclude in the general case we proceed as follows: let $c_{b}(\Omega)$ be the best constant in (3.6). By invariance of (3.6) under the dilation $\delta_{\lambda}$ defined in (3.1) we have,

$$
c_{b}\left(B_{R}\right)=c_{b}\left(B_{1}\right) \text { and } c_{b}\left(B_{R} \backslash\{0\}\right)=c_{b}\left(B_{1} \backslash\{0\}\right) \text { for any } R>0 .
$$

We note that if $B_{R} \backslash\{0\} \subset \Omega \subset \mathbb{R}^{N} \backslash\{0\}$ then,

$$
c_{\beta}^{p}=c_{b}\left(\mathbb{R}^{N} \backslash\{0\}\right) \leq c_{b}(\Omega) \leq c_{b}\left(B_{R} \backslash\{0\}\right)=c_{b}\left(B_{1} \backslash\{0\}\right) .
$$

Let $\phi \in \mathscr{C}_{0}^{\infty}\left(\mathbb{R}^{N} \backslash\{0\}\right)$. Since the support of $\phi$ is compact, then (3.6) holds for $\phi$ with $\Omega=B_{R} \backslash\{0\}, R$ large enough and $c=c_{b}\left(B_{R} \backslash\{0\}\right)=c_{b}\left(B_{1} \backslash\{0\}\right)$. Therefore $c_{b}\left(B_{1} \backslash\{0\}\right) \leq c_{b}\left(\mathbb{R}^{N} \backslash\{0\}\right)$, and from (3.10) we have $c_{b}\left(B_{1} \backslash\{0\}\right)=c_{\beta}^{p}$. Finally, since $B_{R} \subset \Omega \subset \mathbb{R}^{N}$ imply

$$
c_{\beta}^{p} \leq c_{b}\left(\mathbb{R}^{N}\right) \leq c_{b}(\Omega) \leq c_{b}\left(B_{R}\right)=c_{b}\left(B_{1}\right) \leq c_{b}\left(B_{1} \backslash\{0\}\right)=c_{\beta}^{p},
$$

we conclude the proof.

The optimality of the constant $\tilde{c}_{\beta}^{p}$ in (3.8) can be easily proved using the procedure of Remark 2.8.

Other inequalities of Hardy-type related to Baouendi-Grushin operator are given in the following

Theorem 3.5. Let $1 \leq m \leq n$ and let $\beta \in \mathbb{R}$ be fixed. We set $z:=\left(x_{1}, \ldots, x_{m}\right)$.

1. Let $1<p<+\infty$ and let $\Omega \subset \mathbb{R}^{N}$ be an open set. If $m+\beta<0$, we also require that $\Omega \subset\left(\mathbb{R}^{m} \backslash\{0\}\right) \times \mathbb{R}^{N-m}$. Then for every $u \in \mathscr{C}_{0}^{1}(\Omega)$, we have

$$
b_{\beta}^{p} \int_{\Omega}|u|^{p}|z|^{\beta} d \xi \leq \int_{\Omega}\left|\nabla_{\gamma} u\right|^{p}|z|^{p+\beta} d \xi
$$

where $b_{\beta}:=\frac{|m+\beta|}{p}$. 
In particular, for every $u \in \mathscr{C}_{0}^{1}(\Omega)$, we obtain

$$
\left(\frac{|m-p|}{p}\right)^{p} \int_{\Omega} \frac{|u|^{p}}{\llbracket \xi \rrbracket^{p}} d \xi \leq\left(\frac{|m-p|}{p}\right)^{p} \int_{\Omega} \frac{|u|^{p}}{|z|^{p}} d \xi \leq \int_{\Omega}\left|\nabla_{\gamma} u\right|^{p} d \xi .
$$

Moreover, denoting with $B_{r}^{s}$ the Euclidean ball in $\mathbb{R}^{s}$ of radius $r$ and center at the origin, if $B_{r}^{m} \backslash\{0\} \times B_{r}^{N-m} \subset \Omega$ for some $r$, then the constant $b_{\beta}^{p}$ in (3.11) is sharp.

2. Let $p=m>1$. Let $R>0$ and set $\Omega:=\left\{\left(z, x_{m+1} \ldots, x_{n}, y\right) \in \mathbb{R}^{m} \times \mathbb{R}^{n-m} \times\right.$ $\left.\mathbb{R}^{k},|z|<R\right\}$. If $\beta<-1$, then for every $u \in \mathscr{C}_{0}^{1}(\Omega)$, we have

$$
\tilde{b}_{\beta}^{p} \int_{\Omega} \frac{|u|^{p}}{|z|^{p}}\left(\ln \frac{R}{|z|}\right)^{\beta} d \xi \leq \int_{\Omega}\left|\nabla_{\gamma} u\right|^{p}\left(\ln \frac{R}{|z|}\right)^{p+\beta} d \xi,
$$

where $\tilde{b}_{\beta}:=\frac{|\beta+1|}{p}$.

In particular, for every $u \in \mathscr{C}_{0}^{1}(\Omega)$, we obtain

$$
\left(\frac{p-1}{p}\right)^{p} \int_{\Omega} \frac{|u|^{p}}{(|z| \ln (R /|z|))^{p}} d \xi \leq \int_{\Omega}\left|\nabla_{\gamma} u\right|^{p} d \xi .
$$

Moreover, the constant $\tilde{b}_{\beta}^{p}$ is sharp.

Proof. The inequalities (3.11) and (3.13) are a direct consequence of Theorem 2.12. The fact that $\llbracket \xi \rrbracket \geq|z|$ yields the inequality (3.12).

The sharpness of involved constants cannot be proved using the procedure of Remark 2.8. Thus, we shall use a modification of the idea presented in [48].

We prove the optimality of the constant $b_{\beta}^{p}$ in (3.11). The proof of the sharpness of the constant $\tilde{b}_{\beta}^{p}$ is similar.

Let $c_{b}(\Omega)$ be the best constant in (3.11), that is

$$
c_{b}(\Omega):=\inf \left\{\frac{\int_{\Omega}\left|\nabla_{\gamma} \phi\right|^{p}|z|^{\beta+p}}{\int_{\Omega}|\phi|^{p}|z|^{\beta}}, \phi \in \mathscr{C}_{0}^{1}(\Omega), \phi \neq 0\right\} .
$$

From (3.11) we have $c_{b}(\Omega) \geq b_{\beta}^{p}$. We shall prove the equality sign holds.

First we consider the case $\Omega:=\mathbb{R}^{m} \backslash\{0\} \times \mathbb{R}^{n-m} \times \mathbb{R}^{k}$. Observe that if we get the claim for $\Omega$, that is $c_{b}(\Omega)=b_{\beta}^{p}$, from $b_{\beta}^{p} \leq c_{b}\left(\mathbb{R}^{N}\right) \leq c_{b}(\Omega)$, we get the claim also for $\Omega=\mathbb{R}^{N}$.

In what follows $t$ stands for the variables $t:=\left(x_{m+1}, \ldots, x_{n}\right) \in \mathbb{R}^{n-m}$. Let $\phi \in \mathscr{C}_{0}^{1}(\Omega)$ be such that $\phi=u v w$ with $u=u(z) v=v(t), w=w(y), v \in$ $\mathscr{C}_{0}^{1}\left(\mathbb{R}^{m} \backslash\{0\}\right) v \in \mathscr{C}_{0}^{1}\left(\mathbb{R}^{n-m}\right)$ and $w \in \mathscr{C}_{0}^{1}\left(\mathbb{R}^{k}\right)$. It is clear that if $m=n$, then we choose $\phi=u w$ and the following proof results to be slightly simpler. 
By the convexity of the function $\left(q^{2}+r^{2}+s^{2}\right)^{p / 2}$ for $q, r, s \geq 0$ we have

$$
\begin{aligned}
\left(q^{2}+r^{2}+s^{2}\right)^{p / 2} \leq & (1-\lambda-\mu)^{1-p} q^{p}+\lambda^{1-p} r^{p}+\mu^{1-p} s^{p} \\
& \text { for } \lambda, \mu>0, \lambda+\mu<1 .
\end{aligned}
$$

Hence for $\lambda, \mu>0$ such that $\lambda+\mu<1$, we get

$$
\begin{aligned}
\left|\nabla_{\gamma} \phi\right|^{p} & =\left(v^{2} w^{2}\left|\nabla_{z} u\right|^{2}+u^{2} w^{2}\left|\nabla_{t} v\right|^{2}+u^{2} v^{2}\left|\nabla_{\gamma} w\right|^{2}\right)^{p / 2} \\
\leq & (1-\lambda-\mu)^{1-p}|v|^{p}|w|^{p}\left|\nabla_{z} u\right|^{p}+\lambda^{1-p}|u|^{p}|w|^{p}\left|\nabla_{t} v\right|^{p} \\
& \quad+\mu^{1-p}|u|^{p}|v|^{p}\left|\nabla_{\gamma} w\right|^{p} .
\end{aligned}
$$

Therefore, we obtain

$$
\begin{aligned}
c_{b}(\Omega) \leq & \frac{\int_{\Omega}\left|\nabla_{\gamma} \phi\right|^{p}|z|^{\beta+p}}{\int_{\Omega}|\phi|^{p}|z|^{\beta}} \leq(1-\lambda-\mu)^{1-p} \frac{\int_{\Omega}|v|^{p}|w|^{p}\left|\nabla_{z} u\right|^{p}|z|^{\beta+p}}{\int_{\Omega}|v|^{p}|w|^{p}|u|^{p}|z|^{\beta}} \\
& +\lambda^{1-p} \frac{\int_{\Omega}|u|^{p}|w|^{p}\left|\nabla_{t} v\right|^{p}|z|^{\beta+p}}{\int_{\Omega}|u|^{p}|v|^{p}|w|^{p}|z|^{\beta}}+\left.\mu\right|^{1-p} \frac{\int_{\Omega}|u|^{p}|v|^{p}\left|\nabla_{\gamma} w\right|^{p}|z|^{\beta+p}}{\int_{\Omega}|u|^{p}|v|^{p}|w|^{p}|z|^{\beta}} \\
\leq & (1-\lambda-\mu)^{1-p} \frac{\int_{\mathbb{R}^{m}}\left|\nabla_{z} u\right|^{p}|z|^{\beta+p} d z}{\int_{\mathbb{R}^{m}}|u|^{p}|z|^{\beta} d z} \\
& +\lambda^{1-p} \frac{\int_{\mathbb{R}^{n-m}}\left|\nabla_{t} v\right|^{p} d t}{\int_{\mathbb{R}^{n-m}}|v|^{p} d t} \frac{\int_{\mathbb{R}^{m}}|u|^{p}|z|^{\gamma+\beta+p} d z}{\int_{\mathbb{R}^{m}}|u|^{p}|z|^{\beta} d z} \\
& +\mu^{1-p} \frac{\int_{\mathbb{R}^{k}}\left|\nabla_{y} w\right|^{p} d y}{\int_{\mathbb{R}^{k}}|w|^{p} d y} \frac{\int_{\mathbb{R}^{n}}|u|^{p}|v|^{p}|z|^{\beta+p}|x|^{\gamma p} d x}{\int_{\mathbb{R}^{n}}|u|^{p}|v|^{p}|z|^{\beta} d x} .
\end{aligned}
$$

Now, the infimum of the ratio $\int_{\mathbb{R}^{k}}\left|\nabla_{y} w\right|^{p} d y / \int_{\mathbb{R}^{k}}|w|^{p} d y$ vanishes, as well as the ratio $\int_{\mathbb{R}^{n-m}}\left|\nabla_{t} v\right|^{p} d t / \int_{\mathbb{R}^{n-m}}|v|^{p} d t$. From the classical Hardy inequalities (see also Theorem 3.1 with $k=0, n=N=Q$ ), the infimum of $\int_{\mathbb{R}^{m}}\left|\nabla_{z} u\right|^{p}|z|^{\beta+p} d z / \int_{\mathbb{R}^{m}}|u|^{p}|z|^{\beta} d z$ is $b_{\beta}^{p}$. Thus, letting $\lambda, \mu \rightarrow 0$, we get the claim.

In order to complete the proof, we prove the claim in the case $B_{r}^{m} \backslash\{0\} \times$ $B_{r}^{N-m} \subset \Omega \subset \mathbb{R}^{m} \backslash\{0\} \times \mathbb{R}^{N-m}$ for some $r>0$.

Let $B_{r}^{*}:=B_{r}^{m} \backslash\{0\} \times B_{r}^{n-m} \times B_{r^{1+\gamma}}^{k}$. For $s>0$ sufficiently small we have that $B_{s}^{*}=B_{s}^{m} \backslash\{0\} \times B_{s}^{n-m} \times B_{s^{1+\gamma}}^{k} \subset \Omega \subset \mathbb{R}^{m} \backslash\{0\} \times \mathbb{R}^{n-m}$. Thus, we obtain $b_{\beta}^{p}=c_{b}\left(\mathbb{R}^{m} \backslash\{0\} \times \mathbb{R}^{n-m}\right) \leq c_{b}(\Omega) \leq c_{b}\left(B_{s}^{*}\right)$. By invariance of (3.11) under the dilation $\delta_{\lambda}$ defined in (3.1) we have, $c_{b}\left(B_{r}^{*}\right)=c_{b}\left(B_{1}^{*}\right)$ for any $r>0$. Arguing as in the proof of Theorem 3.1, we get the claim and conclude the proof.

\subsection{Heisenberg-Greiner operator}

Let $\xi=(x, y, t) \in \mathbb{R}^{n} \times \mathbb{R}^{n} \times \mathbb{R}, r:=|(x, y)|, \gamma \geq 1$ and let $\mu$ be the matrix defined in (2.3). We remind that for $p=2$ and $\gamma=1 L_{p}$ is the sub-Laplacian $\Delta_{H}$ 
on the Heisenberg-group $\mathbb{H}^{n}$. If $p=2$ and $\gamma=2,3, \ldots, L_{p}$ is a Greiner operator (see [33]).

For $(x, y, t) \in \mathbb{R}^{n} \times \mathbb{R}^{n} \times \mathbb{R}$, we define

$$
N(x, y, t):=\left(\left(x^{2}+y^{2}\right)^{2 \gamma}+t^{2}\right)^{1 / 4 \gamma}=\left(r^{4 \gamma}+t^{2}\right)^{1 / 4 \gamma},
$$

where we have set $r:=\left(x^{2}+y^{2}\right)^{1 / 2}$. Let $Q:=2 n+2 \gamma, p>1$ and let $\Gamma_{p}$ be the function defined as

$$
\Gamma_{p}:=\left\{\begin{array}{l}
N^{\frac{p-Q}{p-1}} \text { if } p \neq Q \\
-\ln N \text { if } p=Q
\end{array} \quad \text { for } \xi \neq 0 .\right.
$$

The function $\Gamma_{p}$ is $L_{p}$ harmonic on $\mathbb{R}^{N} \backslash\{0\}$, that is

$$
-L_{p} \Gamma_{p}=0 \quad \text { on } \mathbb{R}^{N} \backslash\{0\} .
$$

Moreover, arguing as in [2], there exists a constant $l_{p} \neq 0$ such that

$$
-L_{p} \Gamma_{p}=l_{p} \delta_{0}
$$

in weak sense and $l_{p}>0$ if and only if $Q \geq p$ (see also [50]). Moreover, $\left|\nabla_{L} N\right|=$ $\frac{r^{2 \gamma-1}}{N^{2 \gamma-1}}$.

Theorem 3.6. Let $\beta \in \mathbb{R}$ be fixed.

1. Let $1<p<+\infty$ and let $\Omega \subset \mathbb{R}^{N}$ be an open set. If $\beta+Q<0$ we also require that $0 \notin \Omega$. Then, we have

$$
\begin{array}{r}
c_{\beta}^{p} \int_{\Omega}|u|^{p} N^{\beta} \frac{r^{p(2 \gamma-1)}}{N^{p(2 \gamma-1)}} d \xi \leq \int_{\Omega}\left|\nabla_{L} u\right|^{p} N^{p+\beta} d \xi \\
u \in D_{L}^{1, p}\left(\Omega, N^{p+\beta}\right),
\end{array}
$$

where $c_{\beta}:=\frac{|Q+\beta|}{p}$.

In particular, we obtain

$$
\left(\frac{|Q-p|}{p}\right)^{p} \int_{\Omega} \frac{|u|^{p}}{N^{p}} \frac{r^{p(2 \gamma-1)}}{N^{p(2 \gamma-1)}} d \xi \leq \int_{\Omega}\left|\nabla_{L} u\right|^{p} d \xi \quad u \in D_{L}^{1, p}(\Omega) .
$$

Moreover, if $\Omega \cup\{0\}$ is a neighbourhood of the origin, then the constant $c_{\beta}^{p}$ is sharp.

2. Let $p=Q$. Let $R>0$ and set $\Omega:=\left\{\xi \in \mathbb{R}^{N} \mid N(\xi)<R\right\}$. If $\beta<-1$, then we have

$$
\begin{array}{r}
\tilde{c}_{\beta}^{p} \int_{\Omega} \frac{|u|^{p}}{N^{p}}\left(\ln \frac{R}{N}\right)^{\beta} \frac{r^{p(2 \gamma-1)}}{N^{p(2 \gamma-1)}} d \xi \leq \\
\qquad \int_{\Omega}\left|\nabla_{L} u\right|^{p}\left(\ln \frac{R}{N}\right)^{p+\beta} d \xi \\
u \in D_{L}^{1, p}\left(\Omega,(\ln (R / N))^{p+\beta}\right),
\end{array}
$$

where $\tilde{c}_{\beta}:=\frac{|\beta+1|}{p}$. 
In particular, we obtain

$$
\begin{array}{r}
\left(\frac{p-1}{p}\right)^{p} \int_{\Omega} \frac{|u|^{p}}{(N \ln (R / N))^{p}} \frac{r^{p(2 \gamma-1)}}{N^{p(2 \gamma-1)}} d \xi \leq \int_{\Omega}\left|\nabla_{L} u\right|^{p} d \xi \\
u \in D_{L}^{1, p}(\Omega) .
\end{array}
$$

Moreover, the constant $\tilde{c}_{\beta}^{p}$ is sharp.

Remark 3.7. If $\gamma=1$, then the operator $L_{p}$ is the counterpart of the $p$-Laplacian for the sub-Laplacian operator acting on functions defined on the Heisenberg-group $\mathbb{H}^{n}$. In this case the Hardy inequality (3.20) is already obtained for $1<p<Q$ by Garofalo and Lanconelli in [29], Niu, Zhang and Wang in [47]. The author in [19] proves the inequality (3.19) and the sharpness of the involved constant.

In the general case $\gamma \geq 1$, the inequality (3.20) is already obtained in the case $1<p<Q$ for function $u \in \mathscr{C}_{0}^{1}\left(\mathbb{R}^{N} \backslash\{0\}\right)$ in [50].

The proof of the above theorem follows arguing as in the proof of Theorem 3.1. Arguing as in Theorem 3.5 we obtain the following

Theorem 3.8. Let $\beta \in \mathbb{R}$ be fixed.

1. Let $1<p<+\infty$ and let $\Omega \subset \mathbb{R}^{N}$ be an open set. If $2 n+\beta<0$, we also require that $\Omega \subset\left(\mathbb{R}^{2 n} \backslash\{0\}\right) \times \mathbb{R}$. Then for every $u \in \mathscr{C}_{0}^{1}(\Omega)$, we have

$$
b_{\beta}^{p} \int_{\Omega}|u|^{p} r^{\beta} d \xi \leq \int_{\Omega}\left|\nabla_{L} u\right|^{p} r^{p+\beta} d \xi
$$

where $b_{\beta}:=\frac{|2 n+\beta|}{p}$. Moreover, denoting with $B_{r}^{s}$ the Euclidean ball in $\mathbb{R}^{s}$ of radius $r$ with center at the origin, if $B_{r}^{2 n} \backslash\{0\} \times B_{r}^{1} \subset \Omega$ for some $r$, then the constants $b_{\beta}^{p}$ is sharp.

In particular, for every $u \in \mathscr{C}_{0}^{1}(\Omega)$, we obtain

$$
\left(\frac{|2 n-p|}{p}\right)^{p} \int_{\Omega} \frac{|u|^{p}}{N^{p}} d \xi \leq\left(\frac{|2 n-p|}{p}\right)^{p} \int_{\Omega} \frac{|u|^{p}}{r^{p}} d \xi \leq \int_{\Omega}\left|\nabla_{L} u\right|^{p} d \xi .
$$

2. Let $p=2 n$. Let $R>0$ and set $\Omega:=\left\{(x, y, t) \in \mathbb{R}^{2 n} \times \mathbb{R},|(x, y)|<R\right\}$. If $\beta<-1$, then for every $u \in \mathscr{C}_{0}^{1}(\Omega)$, we have

$$
\tilde{b}_{\beta}^{p} \int_{\Omega} \frac{|u|^{p}}{r^{p}}\left(\ln \frac{R}{r}\right)^{\beta} d \xi \leq \int_{\Omega}\left|\nabla_{L} u\right|^{p}\left(\ln \frac{R}{r}\right)^{p+\beta} d \xi,
$$

where $\tilde{b}_{\beta}:=\frac{|\beta+1|}{p}$.

In particular, for every $u \in \mathscr{C}_{0}^{1}(\Omega)$, we obtain

$$
\left(\frac{p-1}{p}\right)^{p} \int_{\Omega} \frac{|u|^{p}}{(|x| \ln (R / r))^{p}} d \xi \leq \int_{\Omega}\left|\nabla_{L} u\right|^{p} d \xi .
$$

Moreover, the constant $\tilde{b}_{\beta}^{p}$ is sharp. 


\subsection{Hardy inequalities on Carnot groups}

In this section we shall present some Hardy inequalities in the framework of Carnot Groups.

We begin by quoting some preliminary facts on these structures and refer the interested reader to $[7,25,26,34])$ for more precise information on this subject.

A Carnot group is a connected, simply connected, nilpotent Lie group $\mathbb{G}$ of dimension $N$ with graded Lie algebra $\mathcal{G}=V_{1} \oplus \cdots \oplus V_{r}$ such that $\left[V_{1}, V_{i}\right]=V_{i+1}$ for $i=1 \ldots r-1$ and $\left[V_{1}, V_{r}\right]=0$. A such integer $r$ is called the step of the group. We set $l=n_{1}=\operatorname{dim} V_{1}, n_{2}=\operatorname{dim} V_{2}, \ldots, n_{r}=\operatorname{dim} V_{r}$. A Carnot group $\mathbb{G}$ of dimension $N$ can be identified, up to an isomorphism, with the structure of a homogeneous Carnot Group $\left(\mathbb{R}^{N}, \circ, \delta_{\lambda}\right)$ defined as follows; we identify $\mathbb{G}$ with $\mathbb{R}^{N}$ endowed with a Lie group law o. We consider $\mathbb{R}^{N}$ splitted in $r$ subspaces $\mathbb{R}^{N}=$ $\mathbb{R}^{n_{1}} \times \mathbb{R}^{n_{2}} \times \cdots \times \mathbb{R}^{n_{r}}$ with $n_{1}+n_{2}+\cdots+n_{r}=N$ and $\xi=\left(\xi^{(1)}, \ldots, \xi^{(r)}\right)$ with $\xi^{(i)} \in \mathbb{R}^{n_{i}}$. We shall assume that there exists a family of Lie group automorphisms, called dilation, $\delta_{\lambda}$ with $\lambda>0$ of the form $\delta_{\lambda}(\xi)=\left(\lambda \xi^{(1)}, \lambda^{2} \xi^{(2)}, \ldots, \lambda^{r} \xi^{(r)}\right)$. The Lie algebra of left-invariant vector fields on $\left(\mathbb{R}^{N}, \circ\right)$ is $\mathcal{G}$. For $i=1, \ldots, n_{1}=l$ let $X_{i}$ be the unique vector field in $\mathcal{G}$ that coincides with $\partial / \partial \xi_{i}^{(1)}$ at the origin. We require that the Lie algebra generated by $X_{1}, \ldots, X_{l}$ is the whole $\mathcal{G}$.

If the above hypotheses are satisfied, we shall call $\mathbb{G}=\left(\mathbb{R}^{N}, \circ, \delta_{\lambda}\right)$ a homogeneous Carnot Group. We denote with $\nabla_{L}$ the vector field $\nabla_{L}:=\left(X_{1}, \ldots, X_{l}\right)^{T}$. The canonical sub-Laplacian on $\mathbb{G}$ is the second-order differential operator defined by $L_{2}:=\Delta_{G}=\sum_{i=1}^{l} X_{i}^{2}$ and we define for $p>1$ the $p$-sub-Laplacian operator $L_{p}(u):=\sum_{i=1}^{l} X_{i}\left(\left|\nabla_{L} u\right|^{p-2} X_{i} u\right)$.

Some important properties of Homogeneous Carnot groups are the following: the Lebesgue measure on $\mathbb{R}^{N}$ coincides with the bi-invariant Haar measure on $\mathbb{G}$. We denote by $Q:=\sum_{i=1}^{r} i n_{i}=\sum_{i=1}^{r} i \operatorname{dim} V_{i}$ the homogeneous dimension of $\mathbb{G}$. For every measurable set $E \subset \mathbb{R}^{N}$, we have $\left|\delta_{\lambda}(E)\right|=\lambda Q_{|E|}$. Since $X_{1}, \ldots, X_{l}$ generate the whole $\mathcal{G}$, the sub-Laplacian $L$ satisfies the Hörmander's hypoellipticity condition. Moreover, the vector fields $X_{1}, \ldots, X_{l}$ are homogeneous of degree 1 with respect to $\delta_{\lambda}$.

A nonnegative continuous function $N: \mathbb{R}^{N} \rightarrow \mathbb{R}_{+}$is called a homogeneous norm on $\mathbb{G}$, if $N\left(\xi^{-1}\right)=N(\xi), N(\xi)=0$ if and only if $\xi=0$ and it is homogeneous of degree 1 with respect to $\delta_{\lambda}$ (i.e. $N\left(\delta_{\lambda}(\xi)\right)=\lambda N(\xi)$ ). A homogeneous norm $N$ defines on $\mathbb{G}$ a pseudo-distance as $d(\xi, \eta):=N\left(\xi^{-1} \eta\right)$. For such a function $d$, there holds only a pseudo-triangular inequality:

$$
d(\xi, \eta) \leq C d(\xi, \zeta)+C d(\zeta, \eta) \quad(\xi, \zeta, \eta \in \mathbb{G})
$$

with $C \geq 1$. Hence, $d$, in general, is not a distance.

If $N$ and $\tilde{N}$ are two homogeneous norms, then they are equivalent, that is, there exists a constant $C>0$ such that $C^{-1} N(\xi) \leq \tilde{N}(\xi) \leq C N(\xi)$.

Let $N$ be a homogeneous norm, then there exists a constant $C>0$ such that $C^{-1}|\xi| \leq N(\xi) \leq C|\xi|^{1 / r}$, for $N(\xi) \leq 1$ and $|\cdot|$ stands for the Euclidean norm. 
An example of homogeneous norm is the following

$$
N_{S}(\xi):=\left(\sum_{i=1}^{r}\left|\xi_{i}\right|^{2 r ! / i}\right)^{1 / 2 r !} .
$$

Notice that if $N$ is a homogeneous norm differentiable a.e., then $\left|\nabla_{L} N\right|$ is homogeneous of degree 0 with respect to $\delta_{\lambda}$, hence $\left|\nabla_{L} N\right|$ is bounded.

Special examples of Carnot groups are the Euclidean spaces $\mathbb{R}^{Q}$. Moreover, if $Q \leq 3$ then any Carnot group is the ordinary Euclidean space $\mathbb{R}^{Q}$.

The most simple nontrivial example of a Carnot group is the Heisenberg-group $\mathbb{H}^{1}=\mathbb{R}^{3}$. For an integer $n \geq 1$, the Heisenberg-group $\mathbb{H}^{n}$ is defined as follows: let $\xi=\left(\xi^{(1)}, \xi^{(2)}\right)$ with $\xi^{(1)}:=\left(x_{1}, \ldots, x_{n}, y_{1}, \ldots, y_{n}\right)$ and $\xi^{(2)}:=t$. We endow $\mathbb{R}^{2 n+1}$ with the group law

$$
\hat{\xi} \circ \tilde{\xi}:=\left(\hat{x}+\tilde{x}, \hat{y}+\tilde{y}, \hat{t}+\tilde{t}+2 \sum_{i=1}^{n}\left(\tilde{x}_{i} \hat{y}_{i}-\hat{x}_{i} \tilde{y}_{i}\right)\right) .
$$

For $i=1, \ldots, n$, consider the vector fields

$$
X_{i}:=\frac{\partial}{\partial x_{i}}+2 y_{i} \frac{\partial}{\partial t}, Y_{i}:=\frac{\partial}{\partial y_{i}}-2 x_{i} \frac{\partial}{\partial t},
$$

and the associated Heisenberg gradient as follows

$$
\nabla_{H}:=\left(X_{1}, \ldots, X_{n}, Y_{1}, \ldots, Y_{n}\right)^{T} .
$$

The sub-Laplacian $\Delta_{H}$ is then the operator defined by

$$
\Delta_{H}:=\sum_{i=1}^{n} X_{i}^{2}+Y_{i}^{2} .
$$

The family of dilation is given by

$$
\delta_{\lambda}(\xi):=\left(\lambda x, \lambda y, \lambda^{2} t\right)
$$

In $\mathbb{H}^{n}$ we can define the canonical homogeneous norm by

$$
|\xi|_{H}:=\left(\left(\sum_{i=1}^{n} x_{i}^{2}+y_{i}^{2}\right)^{2}+t^{2}\right)^{1 / 4} .
$$

The homogeneous dimension is given by $Q=2 n+2$, and the fundamental solution of the sub-Laplacian $-\Delta_{H}$ at point $\eta$ takes the form $\Gamma_{\eta}(\xi)=\left|\eta^{-1} \circ \xi\right|_{H}^{-2 n}$. 
Other particular cases of Carnot groups are the Heisenberg-type groups. They were introduced by Kaplan [36] and have subsequently studied by several authors. We list some properties for Heisenberg-type groups and refer the reader to $[8,30]$ and the reference therein.

Let $\mathbb{G}$ be a Carnot group of step 2 with Lie algebra $\mathcal{G}=V_{1} \oplus V_{2}$ and let $V_{1}$ be endowed with a scalar product $\langle\cdot, \cdot\rangle$. Let $J: V_{2} \rightarrow \operatorname{End}\left(V_{1}\right)$ be defined as

$$
\langle J(\eta) \hat{\xi}, \tilde{\xi}\rangle=\langle[\hat{\xi}, \tilde{\xi}], \eta\rangle, \quad \eta \in V_{2}, \quad \hat{\xi}, \tilde{\xi} \in V_{1} .
$$

We say that $\mathbb{G}$ is of $H$ (eisenberg)-type if for all $\eta \in V_{2}$ we have $J(\eta)^{2}=-|\eta|^{2} I d$.

Let $\mathbb{G}$ be an $H$-type group. Denoting by exp the exponential map exp : $\mathcal{G} \rightarrow \mathbb{G}$ (that is a global diffeomorphism), we define the analytic mappings $x: \mathbb{G} \rightarrow V_{1}$ and $t: \mathbb{G} \rightarrow V_{2}$ by the identity $\xi=\exp (x(\xi)+t(\xi))$. For the sake of simplicity we shall identify $\xi$ with $\xi=(x, t)$.

Let $N$ be defined as

$$
N(\xi):=\left(|x|^{4}+16|t|^{2}\right)^{1 / 4} .
$$

Then $N$ is a homogeneous norm on $\mathbb{G}$. In this setting, the homogeneous dimension is given by $Q=n_{1}+2 n_{2}$ (we remind that $n_{1}=\operatorname{dim} V_{1}$ and $n_{2}=\operatorname{dim} V_{2}$ ).

Let $p>1$ and let $\Gamma_{p}$ be the function defined as

$$
\Gamma_{p}(\xi):=\left\{\begin{array}{l}
N^{\frac{p-Q}{p-1}} \text { if } p \neq Q \\
-\ln N \text { if } p=Q
\end{array} \quad \text { for } \xi \neq 0\right.
$$

The function $\Gamma_{p}$ is $L_{p}$ harmonic on $\mathbb{R}^{N} \backslash\{0\}$, that is

$$
-L_{p} \Gamma_{p}=0 \quad \text { on } \mathbb{R}^{N} \backslash\{0\} .
$$

Moreover, there exists a constant $l_{p}$ such that

$$
-L_{p} \Gamma_{p}=l_{p} \delta_{0}
$$

and if $Q \geq p$, then $l_{p}>0$ (see $\left.[15,35,36]\right)$. Moreover, $\left|\nabla_{L} N(\xi)\right|=\frac{|x|}{N(\xi)}$.

Suppose that a function $u$ has the form $u=u(|x|, t)$, then we have

$$
\left|\nabla_{L} u(\xi)\right|^{2}=\left|\nabla_{x} u\right|^{2}+\frac{|x|^{2}}{4}\left|\nabla_{t} u\right|^{2} \quad \text { and } \quad L u(\xi)=\Delta_{x} u+\frac{|x|^{2}}{4} \Delta_{t} u .
$$

Now we come back to the general Carnot group. It is well-known that there exists a homogeneous norm $N_{2}$ smooth on $\mathbb{G} \backslash\{0\}$ such that $\left(N_{2}(\xi)\right)^{2-Q}$ is a fundamental solution of $-L_{2}$ at 0 (see [25, 27]). On the other hand there exists a homogeneous norm $N_{Q}$ on $\mathbb{G}$ such that $-\ln N_{Q}$ is a fundamental solution of $-L_{Q}$ at 0 (see $[1,35])$. In general these two norms do not agree (see [2]). Moreover, according 
to author's knowledge, the best result on the regularity of $N_{Q}$ is that it is Hölder continuous $([14,15]$, see also [13]).

In spite of lack of information on regularity of $N_{Q}$, we can still use the results of previous section to obtain Hardy inequalities related to $\nabla_{L}$ involving the homogeneous norm $N_{2}$ and $N_{Q}$ for $p=2$ and $p=Q$.

In the case $1<p<Q$ one can argue as follows. Assume that $G_{p}$ is a fundamental solution of $-L_{p}$ at 0 on $\mathbb{G}$ (that is $-L_{p} G_{p}=\delta_{0}$ ) with a singularity at 0 . We set $N_{p}:=G_{p}^{\frac{p-1}{p-Q}}$. Now applying the results of previous section we get a Hardy inequality involving the function $N_{p}$. Using the results presented in [15], it is easy to prove that if $N$ is a homogeneous norm on $\mathbb{G}$, then there exists a constant $C>0$ such that

$$
C N(\xi) \leq N_{p}(\xi) \leq C^{-1} N(\xi) \text { for every } \xi \in \mathbb{G} .
$$

Hence, we obtain a Hardy inequality involving a homogeneous norm $N$, more precisely

Proposition 3.9. Under the above hypotheses, there exists a constant $c>0$ such that for every $u \in \mathscr{C}_{0}^{1}(\mathbb{G})$, we have

$$
c \int_{\mathbb{G}} \frac{|u|^{p}}{N^{p}}\left|\nabla_{L} N_{p}\right|^{p} d \xi \leq \int_{\mathbb{G}}\left|\nabla_{L} u\right|^{p} d \xi .
$$

If, in the previous inequality (3.33) we fix, for instance, $N=N_{2}$, we cannot say anything on the constant $c$ and, in particular, we are not able to estimate $c$ : This is due to the lack of information about the relation between $N_{p}$ and $N_{2}$.

Therefore, in what follows, for $p>1$ we denote with $\Gamma_{p}$ the function defined as

$$
\Gamma_{p}(\xi):=\left\{\begin{array}{ll}
N_{2}^{\frac{p-Q}{p-1}} & \text { if } p \neq Q \\
-\ln N_{2} & \text { if } p=Q
\end{array} \quad \text { for } \xi \neq 0 .\right.
$$

The question if $\Gamma_{p}$ is $L_{p}$ harmonic on $\mathbb{G} \backslash\{0\}$ arises.

In [2] the authors give the following definition

Definition 3.10. The group $\mathbb{G}$ is polarizable if $N_{2}$ is $\infty$-harmonic on $\mathbb{G} \backslash\{0\}$, that is, $N_{2}$ is a solution of

$$
\Delta_{\infty} f:=\frac{1}{2}\left\langle\nabla_{L}\left|\nabla_{L} f\right|^{2}, \nabla_{L} f\right\rangle=0 \quad \text { on } \quad \mathbb{G} \backslash\{0\} .
$$

We recall that for $f \in \mathscr{C}^{2}$ we can write $\Delta_{\infty} f$ also as

$$
\Delta_{\infty} f=\left\langle\left(\nabla_{L}^{2 *} f\right) \nabla_{L} f, \nabla_{L} f\right\rangle
$$

where $\nabla_{L}^{2 *} f$ denotes the symmetrized horizontal Hessian matrix of $f, \nabla_{L}^{2 *} f:=$ $1 / 2\left[\left(\nabla_{L}^{2} f\right)+\left(\nabla_{L}^{2} f\right)^{T}\right]$. 
In [2] the authors prove that if $\mathbb{G}$ is polarizable then $\Gamma_{p}$ defined in (3.34) is $p$-harmonic on $\mathbb{G} \backslash\{0\}$. Moreover, there exists $l_{p} \neq 0$ such that $-L_{p}\left(\Gamma_{p}\right)=l_{p} \delta_{0}$ on $\mathbb{G}$ and $l_{p}>0$ if and only if $1<p \leq Q$.

Actually, the condition that $\Gamma_{p}$ is $L_{p}$-harmonic on $\mathbb{G} \backslash\{0\}$ is also a sufficient condition for the polarizability as specified by the following

Proposition 3.11. The group $\mathbb{G}$ is polarizable if and only if the function $\Gamma_{p}$ defined in (3.34) is $L_{p}$-harmonic on $\mathbb{G} \backslash\{0\}$ for some $p>1, p \neq 2$ (and hence for all $p>1)$.

Proof. The necessary condition is already proved in [2]. Thus, we shall prove the sufficient condition.

Let $u$ be a smooth function. By computation we have

$$
\begin{aligned}
L_{p} u & =\nabla_{L}\left(\left|\nabla_{L} u\right|^{p-2}\right) \cdot \nabla_{L} u+\left|\nabla_{L} u\right|^{p-2} L_{2} u \\
& =(p-2)\left|\nabla_{L} u\right|^{p-4} \Delta_{\infty} u+\left|\nabla_{L} u\right|^{p-2} L_{2} u .
\end{aligned}
$$

Taking into account that $N_{2}^{2-Q}$ is $L_{2}$-harmonic on $\mathbb{G} \backslash\{0\}$, from Lemma 2.18, we have $L_{2} N_{2}=(Q-1) \frac{\left|\nabla_{L} N_{2}\right|^{2}}{N_{2}}$. Hence, applying (3.35) to $N_{2}$ we have

$$
L_{p} N_{2}=(p-2)\left|\nabla_{L} N_{2}\right|^{p-4} \Delta_{\infty} N_{2}+(Q-1) \frac{\left|\nabla_{L} N_{2}\right|^{p}}{N_{2}} .
$$

The thesis will follow if we prove that the identity

$$
L_{p} N_{2}=(Q-1) \frac{\left|\nabla_{L} N_{2}\right|^{p}}{N_{2}}
$$

holds for every $\xi \neq 0$.

Let $p>1, p \neq 2$ be such that $\Gamma_{p}$ is $L_{p}$-harmonic $\mathbb{G} \backslash\{0\}$. First we assume that $p \neq Q$. We apply Lemma 2.18 with $g=N_{2}, \alpha=\frac{p-Q}{p-1}$ to $u=N_{2}$ obtaining the identity (3.37).

Now we consider the case $p=Q$. Since $\Gamma_{Q}=-\ln N_{2}$ is $Q$-harmonic, the function $-\ln \frac{N_{2}}{R}$ is still $Q$-harmonic and positive on $\Omega_{R}:=\left\{\xi \in \mathbb{G} \mid 0<N_{2}(\xi)<\right.$ $R$. Thus applying Lemma 2.18 with $g=-\ln \frac{N_{2}}{R}, \alpha=1$ to $u=N_{2}$ we have that the identity (3.37) is fulfilled on $\Omega_{R}$. Since $R$ is arbitrary we conclude that the identity (3.37) holds on $\mathbb{G} \backslash\{0\}$.

Examples of polarizable Carnot groups are the usual Euclidean space, as well as H-type group and hence the Heisenberg-group. This is proved in [2].

Remark 3.12. Proposition 3.11 provides a straightforward proof of the polarizability of H-type groups. 
Theorem 3.13. Let $p>1$ and let $\Gamma_{p}$ be $L_{p}$-harmonic on $\mathbb{G} \backslash\{0\}$. Let $\beta \in \mathbb{R}$ be fixed and let $N=N_{2}$.

1. Let $1<p<+\infty$ and let $\Omega \subset \mathbb{G}$ be an open set. If $\beta+Q<0$ we also require that $0 \notin \Omega$. Then we have

$c_{\beta}^{p} \int_{\Omega}|u|^{p} N^{\beta}\left|\nabla_{L} N\right|^{p} d \xi \leq \int_{\Omega}\left|\nabla_{L} u\right|^{p} N^{p+\beta} d \xi \quad u \in D_{L}^{1, p}\left(\Omega, N^{p+\beta}\right)$,

where $c_{\beta}:=\frac{|Q+\beta|}{p}$. In particular, we obtain

$$
\left(\frac{|Q-p|}{p}\right)^{p} \int_{\Omega} \frac{|u|^{p}}{N^{p}}\left|\nabla_{L} N\right|^{p} d \xi \leq \int_{\Omega}\left|\nabla_{L} u\right|^{p} d \xi \quad u \in D_{L}^{1, p}(\Omega) .
$$

Moreover, if $\Omega \cup\{0\}$ is a neighbourhood of the origin, then the constant $c_{\beta}^{p}$ is sharp.

2. Let $p=Q>1$. Let $R>0$ and set $\Omega:=\{\xi \in \mathbb{G}, N(\xi)<R\}$. If $\beta<-1$, then we have

$$
\begin{aligned}
\tilde{c}_{\beta}^{p} \int_{\Omega} \frac{|u|^{p}}{N^{p}}\left(\ln \frac{R}{N}\right)^{\beta}\left|\nabla_{L} N\right|^{p} d \xi \leq & \int_{\Omega}\left|\nabla_{L} u\right|^{p}\left(\ln \frac{R}{N}\right)^{p+\beta} d \xi \\
& u \in D_{L}^{1, p}\left(\Omega,(\ln (R / N))^{p+\beta}\right)
\end{aligned}
$$

where $\tilde{c}_{\beta}:=\frac{|\beta+1|}{p}$.

In particular, we obtain

$$
\begin{array}{r}
\left(\frac{p-1}{p}\right)^{p} \int_{\Omega} \frac{|u|^{p}}{(N \ln (R / N))^{p}}\left|\nabla_{L} N\right|^{p} d \xi \leq \int_{\Omega}\left|\nabla_{L} u\right|^{p} d \xi \\
u \in D_{L}^{1, p}(\Omega) .
\end{array}
$$

Moreover, the constant $\tilde{c}_{\beta}^{p}$ is sharp.

Remark 3.14. The above theorem still holds for $p=2$ with $N=N_{2}$ and for $p=$ $Q$ with $N=N_{Q}$ in any Carnot Group and without the hypothesis of polarizability.

If $\mathbb{G}=\mathbb{R}^{N}$ and $\nabla_{L}=\nabla$ is the usual gradient, then $|\nabla N|=1$ and the above inequalities are a generalization of the known Hardy inequalities. results

Let $d_{i}=n_{1}+\cdots+n_{i}$ for $i=1, \ldots, r$ so that $d_{1}=n_{1}=l$ and $d_{r}=N$. It $X_{i}=\frac{\partial}{\partial \xi_{i}}+\sum_{k=1}^{r-1} \sum_{s=d_{k}+1}^{d_{k+1}} P_{i, s, k}\left(\xi_{1}, \ldots, \xi_{d_{1}}, \xi_{d_{1}+1}, \ldots, \xi_{d_{2}}, \ldots, \xi_{d_{k-1}+1}, \ldots, \xi_{d_{k}}\right) \frac{\partial}{\partial \xi_{s}}$,

where $P_{i, s, k}$ is a polynomial homogeneous of degree $k$ with respect to dilation $\delta_{\lambda}$. Denoting with $\mu$ the matrix such that $X_{i}=\sum_{j=1}^{N} \mu_{i j}(\xi) \frac{\partial}{\partial \xi_{j}}$, it results that $\mu$ has the form $\mu=\left(I_{l}, \mu_{1}\right)$, hence in particular $\mu$ has the form (2.29). Therefore we have the following 
Theorem 3.15. Let $1 \leq m \leq l$ and let $\beta \in \mathbb{R}$ be fixed. We set $z:=\left(x_{1}, \ldots, x_{m}\right)$.

1. Let $1<p<+\infty$ and let $\Omega \subset \mathbb{G}$ be an open set. If $m+\beta<0$, we also require that $\Omega \subset\left(\mathbb{R}^{m} \backslash\{0\}\right) \times \mathbb{R}^{N-m}$. Then for every $u \in \mathscr{C}_{0}^{1}(\Omega)$, we have

$$
b_{\beta}^{p} \int_{\Omega}|u|^{p}|z|^{\beta} d \xi \leq \int_{\Omega}\left|\nabla_{L} u\right|^{p}|z|^{p+\beta} d \xi,
$$

where $b_{\beta}:=\frac{|m+\beta|}{p}$. In particular, if $N_{S}$ is the homogeneous norm defined in (3.28), then for every $u \in \mathscr{C}_{0}^{1}(\Omega)$, we obtain

$$
\left(\frac{|m-p|}{p}\right)^{p} \int_{\Omega} \frac{|u|^{p}}{N_{S}^{p}} d \xi \leq\left(\frac{|m-p|}{p}\right)^{p} \int_{\Omega} \frac{|u|^{p}}{|z|^{p}} d \xi \leq \int_{\Omega}\left|\nabla_{L} u\right|^{p} d \xi,
$$

and if $N$ is any homogeneous norm and $p \neq m$, then there exists a constant $c>0$ such that for every $u \in \mathscr{C}_{0}^{1}(\Omega)$, we have

$$
c \int_{\Omega} \frac{|u|^{p}}{N^{p}} d \xi \leq \int_{\Omega}\left|\nabla_{L} u\right|^{p} d \xi
$$

2. Let $p=m>1$. Let $R>0$ and set $\Omega:=\left\{\left(z, \xi_{m+1}, \ldots, \xi_{N}\right) \in \mathbb{R}^{m} \times\right.$ $\left.\mathbb{R}^{N-m},|z|<R\right\}$. If $\beta<-1$, then for every $u \in \mathscr{C}_{0}^{1}(\Omega)$, we have

$$
\tilde{b}_{\beta}^{p} \int_{\Omega} \frac{|u|^{p}}{|z|^{p}}\left(\ln \frac{R}{|z|}\right)^{\beta} d \xi \leq \int_{\Omega}\left|\nabla_{L} u\right|^{p}\left(\ln \frac{R}{|z|}\right)^{p+\beta} d \xi,
$$

where $\tilde{b}_{\beta}:=\frac{|\beta+1|}{p}$. In particular, for every $u \in \mathscr{C}_{0}^{1}(\Omega)$, we obtain

$$
\left(\frac{p-1}{p}\right)^{p} \int_{\Omega} \frac{|u|^{p}}{(|z| \ln (R /|z|))^{p}} d \xi \leq \int_{\Omega}\left|\nabla_{L} u\right|^{p} d \xi .
$$

Moreover, if $\mathbb{G}$ is of $H$-type, $m=l$ and $B_{r}^{l} \backslash\{0\} \times B_{r}^{N-l} \subset \Omega$ for some $r$, then the constants $b_{\beta}^{p}$ and $\tilde{b}_{\beta}^{p}$ in (3.42) and in (3.45) (and hence the constants in (3.43) and (3.46)) are sharp.

Remark 3.16. From the above Theorem 3.15, taking $m=1$, we obtain the inequality (3.44) for any $p>1$, any homogeneous norm $N$ and any function $u \in \mathscr{C}_{0}^{1}(\Omega)$ with $\Omega \subset(\mathbb{R} \backslash\{0\}) \times \mathbb{R}^{N-1}$ and hence also for any smooth function defined on the cone $\mathbb{R}_{+} \times \mathbb{R}^{N-1}$.

Proof. The inequalities (3.42) and (3.45) are a direct consequence of Theorem 2.12. The fact that $N_{S} \geq|z|$ yields the inequality (3.43). Finally the equivalence between homogeneous norms implies (3.44). 
We have to prove the sharpness of the constant in the case $\mathbb{G}$ is of $H$-type with $m=l=\operatorname{dim} V_{1}, z=x$ and $k:=\operatorname{dim} V_{2}$. We prove the optimality of the constant $b_{\beta}^{p}$ in (3.42). The proof for $\tilde{b}_{\beta}^{p}$ in (3.45) is similar.

We shall proceed as in the proof Theorem 3.5, therefore it is sufficient to prove the claim for $\Omega=\left(\mathbb{R}^{m} \backslash\{0\}\right) \times \mathbb{R}^{N-m}$.

Let $c_{b}$ be the best constant in (3.42). We choose $\phi \in \mathscr{C}_{0}^{1}(\Omega)$ such that $\phi=u w$ with $u=u(|x|), w=w(t), v \in \varnothing_{0}^{1}(] 0,+\infty[)$ and $w \in \mathscr{C}_{0}^{1}\left(\mathbb{R}^{k}\right)$.

Arguing as in the proof of Theorem 3.5, using the convexity of the function $\left(r^{2}+s^{2}\right)^{p / 2}$, and the fact that

$$
\left|\nabla_{L} \phi\right|^{2}=\left|\nabla_{x} \phi\right|^{2}+\frac{|x|}{4}\left|\nabla_{t} \phi\right|^{2}=w^{2}\left(u^{\prime}(|x|)\right)^{2}+\frac{|x|}{4} u^{2}\left|\nabla_{t} w\right|^{2},
$$

we obtain for $0<\lambda<1$

$$
\begin{aligned}
c_{b} \leq & (1-\lambda)^{1-p} \frac{\int_{\mathbb{R}^{l}}\left|\nabla_{x} u\right|^{p}|x|^{\beta+p} d x}{\int_{\mathbb{R}^{l}}|u|^{p}|x|^{\beta} d x} \\
& +\lambda^{1-p} \frac{\int_{\mathbb{R}^{k}}\left|\nabla_{t} w\right|^{p} d t}{\int_{\mathbb{R}^{k}}|w|^{p} d t} \frac{\int_{\mathbb{R}^{l}}|u|^{p}|x|^{\gamma+\beta+p}}{\int_{\mathbb{R}^{l}}|u|^{p}|x|^{\beta}} .
\end{aligned}
$$

The infimum of $\int_{\mathbb{R}^{k}}\left|\nabla_{t} w\right|^{p} d t / \int_{\mathbb{R}^{k}}|w|^{p} d t$ vanishes. The infimum of

$$
\frac{\int_{\mathbb{R}^{l}}\left|\nabla_{x} u\right|^{p}|x|^{\gamma+\beta+p}}{\int_{\mathbb{R}^{l}}|u|^{p}|x|^{\beta}}=\frac{\int_{0}^{+\infty}\left|u^{\prime}(s)\right| s^{\beta+p+l-1} d s}{\int_{0}^{+\infty}|u(s)| s^{\beta+l-1} d s}
$$

is $b_{\beta}^{p}$. Indeed, it follows from Theorem 3.13 with $Q=1$ and $\beta$ replaced by $\beta+l-1$. Letting $\lambda \rightarrow 0$ in (3.47), we conclude the proof.

The next results deal with Hardy inequalities for functions defined on a ball or on the complement of a ball and involving the distance from the boundary.

If $\mathbb{G}$ is the Euclidean space or an $H$-type group, then the pseudo-distance $d_{2}(\xi, \eta):=N_{2}\left(\xi^{-1} \eta\right)$ is actually a distance (see [17]). In a general Carnot group, there holds only the pseudo-triangular inequality (3.27). Hence, $d_{2}$, in general is not a distance. Therefore, in the general framework we shall deal with the CarnotCarathéodory distance $d_{C C}$, defined as follows. Let $\gamma:[a, b] \rightarrow \mathbb{R}^{N}$ be a piecewise smooth curve, we call $\gamma$ a horizontal path if $\dot{\gamma}(t)$ belongs to $V_{1}$ whenever it exists. Then for every $\xi, \eta \in \mathbb{G}$, we define

$$
d_{C C}(\xi, \eta):=\inf \left\{\int|\dot{\gamma}|, \gamma:[a, b] \rightarrow \mathbb{R}^{N} \text { horizontal path with } \gamma(a)=\xi, \gamma(b)=\eta\right\} \text {. }
$$

In the framework of Carnot group, by Chow Theorem, for every $\xi, \eta \in \mathbb{G}$, it results $d_{C C}(\xi, \eta)<\infty$, and hence $d_{C C}$ is a metric on $\mathbb{G}$. The distance $d_{C C}$ is left invariant 
with respect to the group action and it is homogeneous of degree 1 with respect to dilation $\delta_{\lambda}$, namely

$$
d_{C C}(\zeta \xi, \zeta \eta)=d_{C C}(\xi, \eta), \quad d_{C C}\left(\delta_{\lambda}(\xi), \delta_{\lambda}(\eta)\right)=\lambda d_{C C}(\xi, \eta) \quad \zeta, \eta, \xi \in \mathbb{G}, \lambda>0 .
$$

Hence, $d_{C C}(\cdot, 0)$ is a homogeneous norm.

Theorem 3.17. Let $p>1$ and let $\Gamma_{p}$ be $L_{p}$-harmonic on $\mathbb{G} \backslash\{0\}$. Let $R>0$ and set $\Omega:=\left\{\xi \in \mathbb{G}, N_{2}(\xi)<R\right\}$. We have

$$
\left(\frac{p-1}{p}\right)^{p} \int_{\Omega} \frac{|u|^{p}}{\left(R-N_{2}\right)^{p}}\left|\nabla_{L} N_{2}\right|^{p} d \xi \leq \int_{\Omega}\left|\nabla_{L} u\right|^{p} d \xi, \quad u \in D_{L}^{1, p}(\Omega) .
$$

The constant $\left(\frac{p-1}{p}\right)^{p}$ is optimal.

Moreover, we have

$$
c^{p} \int_{\Omega} \frac{|u|^{p}}{\delta^{p}}\left|\nabla_{L} N_{2}\right|^{p} d \xi \leq \int_{\Omega}\left|\nabla_{L} u\right|^{p} d \xi, \quad u \in D_{L}^{1, p}(\Omega)
$$

where $\delta$ is one of the following functions

a) $\delta(\xi):=d_{C C}(\xi, \partial \Omega):=\inf \left\{d_{C C}(\xi, \eta), \eta \in \partial \Omega\right\}, c:=\frac{p-1}{p} \frac{1}{C_{2}}$ and $C_{2}:=$ $\left\|\nabla_{L} N_{2}\right\|_{L^{\infty}}$;

or

b) $\delta(\xi):=d_{2}(\xi, \partial \Omega):=\inf \left\{d_{2}(\xi, \eta), \eta \in \partial \Omega\right\}$ and $c:=\frac{p-1}{p}$ provided $d_{2}(\xi, \eta):=$ $N_{2}\left(\xi^{-1} \eta\right)$ is a distance.

Remark 3.18. The constant $c^{p}$ in (3.51) with this generality cannot be improved. Indeed, if $\mathbb{G}$ is the Euclidean space $\mathbb{R}^{N}$ and $\nabla_{L}=\nabla$, we have $c=\frac{p-1}{p}$, which is the best constant (see [41]).

Proof. From (3.36), and if $p \neq 2$ by polarizability of $\mathbb{G}$, we get

$$
L_{p} N_{2}=\left|\nabla_{L} N_{2}\right|^{p-2} L_{2} N_{2}=(Q-1) \frac{\left|\nabla_{L} N_{2}\right|^{p}}{N_{2}} \geq 0 .
$$

Therefore, choosing $d(\xi)=R-N_{2}(\xi)$, we are in the position to apply Theorem 2.7 and from (2.19) we get (3.50).

Applying the scheme outlined in Remark 2.8, we obtain the optimality of the constant.

We prove the inequality (3.51). Let $\delta=d_{C C}(\cdot, \partial \Omega)$ or $\delta=d_{2}(\cdot, \partial \Omega)$, let $\xi \in \Omega$ be fixed and let $\tau \in \partial \Omega$ be a point where the minimum is attained, that is $\delta(\xi)=d_{C C}(\tau, \xi)$ or $\delta(\xi)=d_{2}(\tau, \xi)$.

First we prove the inequality (3.51) in the case b). The inequality (3.51) follows from (3.50) and the fact that $d_{2}(\cdot, \cdot)$ is a distance. By triangular inequality, we have 
$R=d_{2}(\tau, 0) \leq d_{2}(\tau, \xi)+d_{2}(\xi, 0)=\delta(\xi)+N_{2}(\xi)$, and hence we get the inequality (3.51).

We prove the case a). By the inequality

$$
\left|N_{2}(\xi)-N_{2}(\eta)\right| \leq\left\|\nabla_{L} N_{2}\right\|_{L^{\infty}} d_{C C}(\xi, \eta), \quad \text { for every } \xi, \eta \in \mathbb{G},
$$

we have

$$
R-N_{2}(\xi)=N_{2}(\tau)-N_{2}(\xi) \leq C_{2} d_{C C}(\tau, \xi)=C_{2} \delta(\xi),
$$

which concludes the proof.

Remark 3.19. We remark that if $\phi$ is a regular H-convex function and $\nabla_{L} \phi \neq 0$ a.e., then for $p \geq 2$, from (3.35), we have $-L_{p}(\phi) \leq 0$, thus in order to obtain Hardy inequalities involving the function $\phi$ we can apply the results of previous section. For H-convex function on Carnot groups, we refer the interested reader to $[22,38,39]$. For instance, in [22] the authors prove that in an H-type group the gauge $N$ defined in (3.29) is H-convex, hence $R-N(\xi)$ is H-concave and we can obtain again the inequalities (3.50) and (3.51).

We conclude with a Hardy inequality on an exterior domain.

Theorem 3.20. Let $p>Q$ and let $\Gamma_{p}$ be $L_{p}$-harmonic on $\mathbb{G} \backslash\{0\}$. Let $R>0$ and set $\Omega:=\left\{\xi \in \mathbb{G}, N_{2}(\xi)>R\right\}$. For every $u \in \mathscr{C}_{0}^{1}(\Omega)$ we have

$$
\left(\frac{|p-Q|}{p}\right)^{p} \int_{\Omega} \frac{|u|^{p}}{\left(N_{2}-R\right)^{p}}\left|\nabla_{L} N_{2}\right|^{p} d \xi \leq \int_{\Omega}\left|\nabla_{L} u\right|^{p} d \xi
$$

Moreover, for every $u \in \mathscr{C}_{0}^{1}(\Omega)$, we have

$$
c^{p} \int_{\Omega} \frac{|u|^{p}}{\delta^{p}}\left|\nabla_{L} N_{2}\right|^{p} d \xi \leq \int_{\Omega}\left|\nabla_{L} u\right|^{p} d \xi
$$

where $\delta$ is one of the following functions

a) $\delta(\xi):=d_{C C}(\xi, \partial \Omega):=\inf \left\{d_{C C}(\xi, \eta), \eta \in \partial \Omega\right\}, c:=\frac{|p-Q|}{p} \frac{1}{C_{2}}$ and $C_{2}:=$ $\left\|\nabla_{L} N_{2}\right\|_{L^{\infty}}$;

or

b) $\delta(\xi):=d_{2}(\xi, \partial \Omega):=\inf \left\{d_{2}(\xi, \eta), \eta \in \partial \Omega\right\}$ and $c:=\frac{|p-Q|}{p}$ provided $_{2}(\xi, \eta):=$ $N_{2}\left(\xi^{-1} \eta\right)$ is a distance.

Proof. Let $d$ be defined as $d(\xi):=N_{2}(\xi)^{\frac{p-Q}{p-1}}-R^{\frac{p-Q}{p-1}}(\xi \in \Omega)$. It is clear that $d$ is positive and $L_{p} d=0$. Applying Theorem 2.7 we derive

$$
\left(\frac{|p-Q|}{p}\right)^{p} \int_{\Omega}|u|^{p}\left(\frac{N_{2}^{\frac{1-Q}{p-1}}}{N_{2}(\xi)^{\frac{p-Q}{p-1}}-R^{\frac{p-Q}{p-1}}}\right)^{p}\left|\nabla_{L} N_{2}\right|^{p} d \xi \leq \int_{\Omega}\left|\nabla_{L} u\right|^{p} d \xi .
$$


It is easy to check that for $\xi \in \Omega$, it results

$$
N_{2}(\xi)^{\frac{p-Q}{p-1}}-R^{\frac{p-Q}{p-1}} \leq N_{2}^{\frac{1-Q}{p-1}}\left(N_{2}-R\right),
$$

which with (3.54) implies (3.52).

Arguing as in the proof of Theorem 3.17, we obtain the missing inequality (3.53).

Remark 3.21. The constant $c^{p}$ in (3.52) and (3.53) cannot be improved in this generality. Indeed if $\nabla_{L}$ is the usual gradient $\nabla$, then this constant is sharp (see [42]).

\section{References}

[1] Z. M. Balogh, I. Holopainen and J. T. Tyson, Singular solutions, homogeneous norms, and quasiconformal mappings in Carnot groups, Math. Ann. 324 (2002), 159-186.

[2] Z. M. BALOGH and J. T. Tyson, Polar coordinates in Carnot groups, Math. Z. 241 (2002), 697-730.

[3] P. BARAS and J. A. Goldstein, The heat equation with a singular potential, Trans. Amer. Math. Soc. 284 (1984), 121-139.

[4] G. Barbatis, S. FilipPas and A. Tertikas, Series expansion for $L^{p}$ Hardy inequalities, Indiana Univ. Math. J. 52 (2003), 171-190.

[5] G. Barbatis, S. FilipPas and A. Tertikas, A unified approach to improved L $^{p}$ Hardy inequalities with best constants, Trans. Amer. Math. Soc. 356 (2004), 2169-2196.

[6] T. BIESKE and J. Gong, The P-Laplace Equation on a class of Grushin-type Spaces, Proc. Amer. Math. Soc., to appear.

[7] A. Bonfiglioli and F. Uguzzoni, A Note on Lifting of Carnot groups, Rev. Mat. Iberoamericana. To appear.

[8] A. Bonfiglioli and F. Uguzzoni, Nonlinear Liouville theorems for some critical problems on H-type groups, J. Funct. Anal. 207 (2004), 161-215.

[9] H. BREZIS and X. CABRÉ, Some simple nonlinear PDE's without solutions, Boll. Unione Mat. Ital. Sez. B Artic. Ric. Mat. (8) 1 (1998), 223-262.

[10] H. BRezIS and M. MARCus, Hardy's inequalities revisited, Ann. Scuola Norm. Sup. Pisa Cl. Sci. (4) 25 (1997), 217-237 (1998). Dedicated to Ennio De Giorgi.

[11] H. BREZIS, M. MARCUS and I. SHAFRIR, Extremal functions for Hardy's inequality with weight, J. Funct. Anal. 171 (2000), 177-191.

[12] H. BREzis and J. L. VÁzQUez, Blow-up solutions of some nonlinear elliptic problems, Rev. Mat. Univ. Complut. Madrid 10 (1997), 443-469.

[13] L. CAPOGNA, Regularity for quasilinear equations and 1-quasiconformal maps in Carnot groups, Math. Ann. 313 (1999), no. 2, 263-295.

[14] L. Capogna, D. Danielli and N. Garofalo, An embedding theorem and the Harnack inequality for nonlinear subelliptic equations, Comm. Partial Differential Equations 18 (1993), 1765-1794.

[15] L. Capogna, D. Danielli and N. Garofalo, Capacitary estimates and the local behavior of solutions of nonlinear subelliptic equations, Amer. J. Math. 118 (1996), 1153-1196.

[16] G. CARRon, Inégalités de Hardy sur les variétés riemanniennes non-compactes, J. Math. Pures Appl. (9) 76 (1997), 883-891.

[17] J. Cygan, Subadditivity of homogeneous norms on certain nilpotent Lie groups, Proc. Amer. Math. Soc. 83 (1981), 69-70. 
[18] L. D’ Ambrosio, Hardy inequalities related to Grushin type operators, Proc. Amer. Math. Soc. 132 (2004), 725-734.

[19] L. D'Ambrosio, Some Hardy Inequalities on the Heisenberg Group, Differential Equations 40 (2004), 552-564.

[20] L. D'Ambrosio and S. LuCENTE, Nonlinear Liouville theorems for Grushin and Tricomi operators, J. Differential Equations 193 (2003), 511-541.

[21] L. D'Ambrosio, E. Mitidieri and S. I. Pohozaev, Representation Formulae and Inequalities for Solutions od a Class of Second Order Partial Differential Equations, Trans. Amer. Math. Soc. (2005), PII S 0002-9947(05)03717-7.

[22] D. Danielli, N. Garofalo and D.-M. Nhieu, Notions of convexity in Carnot groups, Comm. Anal. Geom. 11 (2003), 263-341.

[23] E. B. DAviEs, The Hardy constant, Q. J. Math. (2) 46 (1995), 417-431.

[24] E. B. Davies and A. M. HinZ, Explicit constants for Rellich inequalities in $L_{p}(\Omega)$, Math. Z. 227 (1998), 511-523.

[25] G. B. Folland, Subelliptic estimates and function spaces on nilpotent Lie groups, Ark. Mat. 13 (1975), 161-207.

[26] G. B. Folland and E. M. Stein, “ Hardy spaces on homogeneous groups”, volume 28 of Mathematical Notes, Princeton University Press, Princeton, N.J., 1982.

[27] L. Gallardo, Capacités, mouvement brownien et problème de l'épine de Lebesgue sur les groupes de Lie nilpotents, In: "Probability measures on groups", Oberwolfach, 1981, volume 928 of Lecture Notes in Math., Springer, Berlin, pp. 96-120.

[28] J. P. García Azorero and I. Peral Alonso, Hardy inequalities and some critical elliptic and parabolic problems, J. Differential Equations 144 (1998), 441-476.

[29] N. Garofalo and E. LANCONELli, Frequency functions on the Heisenberg group, the uncertainty principle and unique continuation, Ann. Inst. Fourier (Grenoble) 40 (1990), 313-356.

[30] N. Garofalo and D. VAssileV, Symmetry properties of positive entire solutions of Yamabe-type equations on groups of Heisenberg type, Duke Math. J. 106 (2001), 411-448.

[31] F. Gazzola, H.-C. Grunau and E. Mitidieri, Hardy inequalities with optimal constants and remainder terms, Trans. Amer. Math. Soc. 356 (2004), 2149-2168.

[32] J. A. Goldstein and Q. S. ZHANG, On a degenerate heat equation with a singular potential, J. Funct. Anal. 186 (2001), 342-359.

[33] P. C. GREINER, A fundamental solution for a nonelliptic partial differential operator, Canad. J. Math. 31 (1979), 1107-1120.

[34] J. Heinonen, Calculus on Carnot groups, In: "Fall School in Analysis" (Jyväskylä, 1994), volume 68 of Report . Univ. Jyväskylä, Jyväskylä, pp. 1-31.

[35] J. Heinonen and I. Holopainen, Quasiregular maps on Carnot groups, J. Geom. Anal. 7 (1997), 109-148.

[36] A. KAPLAN, Fundamental solutions for a class of hypoelliptic PDE generated by composition of quadratic forms, Trans. Amer. Math. Soc. 258 (1980), 147-153.

[37] I. KoмBE, Nonlinear degenerate parabolic equations for Baouendi-Grushin operators (2004), preprint.

[38] G. Lu, J. Manfredi and B. Stroffolini, Convex functions on the Heisenberg group, Calc. Var. Partial Differential Equations 19 (2004), 1-22.

[39] V. Magnani, Lipschitz continuity, Aleksandrov theorem and characterizations for $\mathrm{H}$ convex function (2003). URL http://cvgmt.sns.it/papers/mag03a, Preprint.

[40] M. Marcus, V. J. Mizel and Y. Pinchover, On the best constant for Hardy's inequality in $\mathbf{R}^{n}$, Trans. Amer. Math. Soc. 350 (1998), 3237-3255.

[41] T. MATsKewich and P. E. SobolevsKiI, The best possible constant in generalized Hardy's inequality for convex domain in $\mathbf{R}^{n}$, Nonlinear Anal. 28 (1997), 1601-1610.

[42] T. MatsKewich and P. E. SobolevsKiI, The sharp constant in Hardy's inequality for complement of bounded domain, Nonlinear Anal. 33 (1998), 105-120. 
[43] V. G. MAZ’JA, "Sobolev spaces", Springer Series in Soviet Mathematics. Springer-Verlag, Berlin 1985. Translated from the Russian by T. O. Shaposhnikova.

[44] E. MiTIDIERI, A simple approach to Hardy inequalities, Mat. Zametki 67 (2000), 563-572.

[45] E. Mitidieri and S. I. PoHOZAEV, Nonexistence of weak solutions for some degenerate elliptic and parabolic problems on $\mathbf{R}^{\mathbf{n}}$, J. Evol. Equ. 1 (2001), 189-220.

[46] E. Mitidieri and S. I. POHOZAEV, A priori estimates and blow-up of solutions of nonlinear partial differential equations and inequalities, Proc. Steklov Inst. Math. 234 (2001), $1-362$.

[47] P. NiU, H. Zhang and Y. WAng, Hardy-type and Rellich type inequalities on the Heisenberg group, Proc. Amer. Math. Soc. 129 (2001), 3623-3630.

[48] S. SeCCHI, D. SMets and M. Willem, Remarks on a Hardy-Sobolev inequality, C. R. Math. Acad. Sci. Paris 336 (2003), 811-815.

[49] J. L. VAZQUEZ and E. ZUAZUA, The Hardy inequality and the asymptotic behaviour of the heat equation with an inverse-square potential, J. Funct. Anal. 173 (2000), 103-153.

[50] H. ZHANG and P. NIU, Hardy-type inequalities and Pohozaev-type identities for a class of p-degenerate subelliptic operators and applications, Nonlinear Anal. 54 (2003), 165-186.

Dipartimento di Matematica

Via E. Orabona, 4

I-70125 Bari, Italy

dambros@dm.uniba.it 